\title{
Development and validation of an ultra-high sensitive next-generation sequencing assay for molecular diagnosis of clinical oncology
}

\author{
JIAO LIANG $^{1 *}$, YAOGUANG SHE ${ }^{2 *}$, JIAQI ZHU ${ }^{3 *}$, LONGGANG WEI $^{3}$, LANYING ZHANG $^{3}$, \\ LIANJU GAO ${ }^{3}$, YAN WANG ${ }^{3}$, JING XING $^{3}$, YANG GUO ${ }^{3}$, XUEHONG MENG $^{3}$ and PEIYU LI $^{2}$
}

${ }^{1}$ State Key Laboratory of Biomembrane and Membrane Biotechnology, School of Medicine, National Engineering
Laboratory for Anti-tumor Therapeutics, Tsinghua University, Beijing 100084; ${ }^{2}$ Department of General Surgery,
Chinese PLA General Hospital, Beijing $100853 ;{ }^{3}$ Novogene Bioinformatics Institute, Beijing 100083, P.R. China

Received June 8, 2016; Accepted September 7, 2016

DOI: 10.3892/ijo.2016.3707

\begin{abstract}
Dramatic improvements in the understanding of oncogenes have spurred the development of molecular target therapies, which created an exigent need for comprehensive and rapid clinical genotyping. Next-generation sequencing (NGS) assay with increased performance and decreased cost is becoming more widely used in clinical diagnosis. However, the optimization and validation of NGS assay remain a challenge, especially for the detection of somatic variants at low mutant allele fraction (MAF). In the present study, we developed and validated the Novogene Comprehensive Panel (NCP) based on targeted capture for NGS analysis. Due to the high correlation between SNV/INDEL detection performance and target coverage, here we focused on these two types of variants for our deep sequencing strategy. To validate the capability of NCP in single-nucleotide variant (SNV) and small insert and deletion (INDEL) detection, we implemented a practical validation strategy with pooled cell lines, deep sequencing of pooled samples ( $>2000 \mathrm{X}$ average unique coverage across target region) achieving $>99 \%$ sensitivity and high specificity (positive predictive value, PPV $>99 \%$ ) for all types of variations with expected MAF $>5 \%$. Furthermore, given the high sensitivity and that false positive may exist in this assay, we confirmed its accuracy of variants with MAF $<5 \%$ using
\end{abstract}

Correspondence to: Dr Xuehong Meng, Beijing Novogene Bioinformatics Technology, Co., Ltd., Jinma Building, No. 38 Xueqing Road, Haidian, Beijing, P.R. China

E-mail: mengxuehong@novogene.com

Dr Peiyu Li, Department of General Surgery, Chinese PLA General Hospital, Beijing 100853, P.R. China

E-mail: peiyuli301@163.com

${ }^{*}$ Contributed equally

Key words: next-generation sequencing, target therapy, sensitivity, deep sequencing, clinical
35 formalin-fixed and paraffin-embedded (FFPE) tumor specimens by QuantStudio 3D Digital PCR (dPCR; Life Technologies) and obtained a high consistency (32 of 35 mutations detected by NGS were verified). We also used the amplification refractory mutation system (ARMS) to verify the variants with a MAF in a broad range of 2-63\% detected in 33 FFPE samples and reached a $100 \%$ PPV for this assay. As a potential clinical diagnosis tool, NCP can robustly and comprehensively analyze clinical-related genes with high sensitivity and low cost.

\section{Introduction}

Cancer is a genomic disease harboring a cocktail of mutated genes. Personalized medicine approaches based on molecular studies and cytogenetic analysis can treat with therapies directly on mutated cancer driving genes (1-4). For example, crizotinib (PF-02341066), a small-molecular inhibitor of the anaplastic lymphoma kinase (ALK), and kinase inhibitor vemurafenib (PLX4032) against BRAF (5-7), both have dramatic effects on most patients with corresponding driver mutations. In fact, hundreds of frequent somatic mutations, which involved in multiple cellular pathways, have been identified in different types of cancer during the past decades (8), and more comprehensive diagnostic approaches are needed to identify the individual driver mutations which have important impact on tumor progression in different cancer patients (9) and thus, could serve as therapeutic targets in clinical treatment. To assess the status of these biomarkers, several approaches have been implemented in clinical diagnosis, such as fluorescence in situ hybridization (FISH), immunohistochemistry (IHC) and Sanger methodology (10-13). However, due to the high cost and technical limitations, it is unaffordable to do the multiplexed assessment of driving somatic alterations.

NGS has already been used to identify hundreds of driving mutations and analyze tens of thousands of tumor samples in a high-throughput with increased performance and decreased costs (14-16), which makes it possible to serve as a clinical testing approach. In reality, commercial NGS-based assays have already been developed and validated to provide 
comprehensive genomic test in clinic (17-20). These assays usually have a good performance when detecting variants with high mutant allele frequencies (MAF $>10 \%)$. However, variants with low MAF usually appear in tumor tissues for many reasons, including contaminating normal cells and intra-tumor heterogeneity $(21,22)$. Therefore, it is critical to develop a robust clinical assay that can detect low allele frequency mutations. Here we developed an ultra-high sensitive NGS-based assay, which interrogates all 7011 exons of 483 cancer-related genes and 94 introns of 18 genes with re-arrangement. Using the Illumina HiSeq X platform, hybridization-based capture of target regions reached a high-coverage $(>2000 \mathrm{X})$ with acceptable cost. With in-house data analysis approaches, we could identify low MAF $(0.5 \%)$ variants from sequencing error accurately. We used pools of mixed cell lines with known alterations to perform analytical validation, and 35 FFPE tissue samples to confirm the specificity of low MAF variants detection performance in clinic by dPCR (23). In addition, ARMS-PCR (24) was used to confirm the overall specificity of our assay.

\section{Materials and methods}

NCP NGS design. Novo assay was developed to characterize SNV/INDEL, CNV and gene fusion in 483 cancer-related genes. These genes were selected based on My Cancer Genome database (https://www.mycancergenome.org), Catalogue of Somatic Mutations in Cancer (COSMIC) and other sources $(18,25)$. Briefly, genes containing clinically important variants and genes have been reported as cancer-related were included based on a record of reimbursement in sequencing. All exons of these genes were considered which underwent hybridization-based capture from 483 cancer-related genes (Table I). For structural rearrangements detection, introns spanning recurrent fusion breakpoints were also included. Agilent's proprietary algorithm and synthetic process was used to generate the baits. The hybrid selection was done using a pool of 120-mer RNA-based baits (Agilent SureSelect) with overlap excess 3-fold for target region. All 47660 hybrid baits for catching target region constitute $2.3 \mathrm{Mb}$ genomic positions, including 7011 exons and 94 introns.

Clinical specimens. Tumor specimens were collected from non-small cell lung cancer (NSCLC) and breast cancer patients at Chinese PLA General Hospital with informed consent according to the internal Review and rules of Ethics. In the very beginning of this assay, clinical samples should match several standards as follows to ensure downstream analysis. At least 10 slices of $5 \mu \mathrm{m}$ FFPE sections or tissues with a volume of $>1$ was required. For each sample, hematoxylineosin stained slides (Fig. 1) were prepared and reviewed by a pathologist to estimate tumor purity. All samples with $<50 \%$ tumor purity were marked for tumor enrichment by microdissection to minimize contamination from normal cells (Fig. 2).

Cell line sample collection. Normal cell lines harboring the population distribution of known germ line variants were mixed, and multiplexed pools with low MAF variants were used to assess and validate the limit of variant detection. First of all, to get the variants set for assessment, we sequenced 5 cell

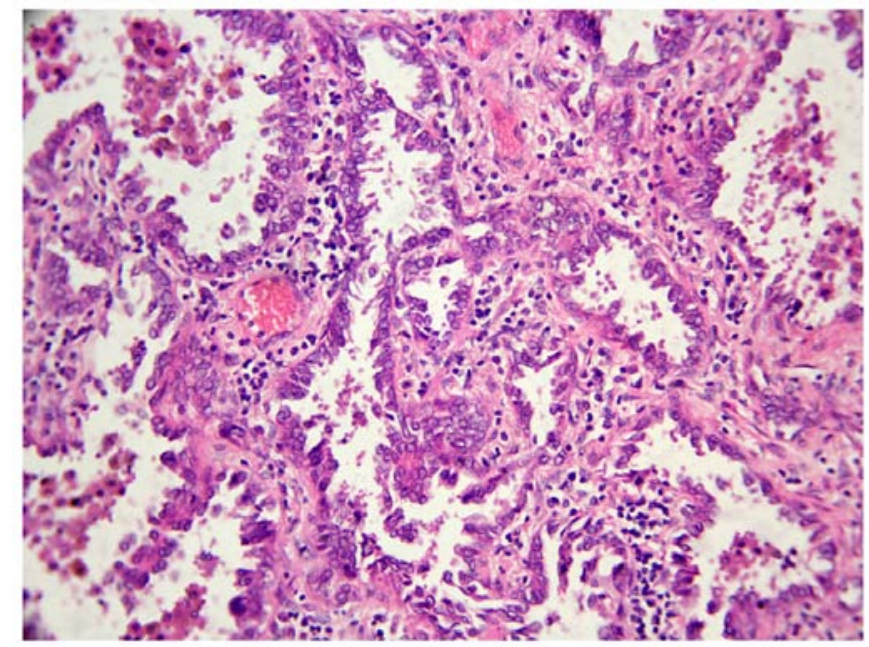

Figure 1. Example of H\&E stained FFPE sample. H\&E stained FFPE sample for sample with id 7 in 3D digital PCR test.

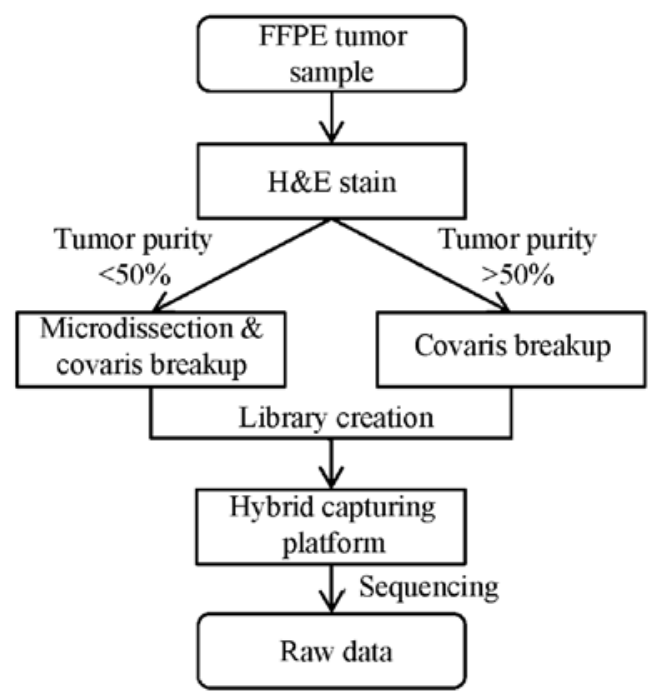

Figure 2. DNA extraction and library preparation. DNA extracted from spliced FFPE tumor sample prepared for sequencing.

lines from the 1000 Genomes Project (26) individually and got the SNP and INDEL sites from dbSNP database (build 146) consistent with a homozygous (MAF $>90 \%$ ) or heterozygous $(40 \%<\mathrm{MAF}<60 \%)$. To estimate the INDEL detection performance, 3 additional cell lines from COSMIC database (http:// cancer.sanger.ac.uk/cancergenome/projects/cosmic/) which also were sequenced individually to get the original MAF of cancer-related somatic variants in each sample. All 8 cell lines were mixed together in designed proportions, and the expected MAF of each variant was calculated on the mixed ratios (Table II). Eventually, we achieved the 2625 variants spanning a range of expected MAF (0.5-20\%) and INDEL lengths (1-40 base pair, bp) as gold-standard (Table III). Cell lines obtained from Coriell Institute (http://ccr.coriell.org/) and ATCC (http://www.atcc.org/) were routinely cultured in Dulbecco's modified Eagle's media (DMEM) with $10 \%$ heat-inactivated fetal bovine serum (FBS; Invitrogen, Waltham, MA, USA) in a $75-\mathrm{cm}^{2}$ cell culture flask. The cells were seeded into cell 
Table I. Genes and transcripts ID targeted in hybridization capture.

\begin{tabular}{|c|c|c|c|c|c|}
\hline Gene symbol & Transcripts ID & Gene symbol & Transcripts ID & Gene symbol & Transcripts ID \\
\hline ABCB1 & NM_000927 & ETV6 & NM_001987 & NUP93 & NM_001242796 \\
\hline $\mathrm{ABCC} 1$ & NM_004996 & EWSR 1 & NM_001163287 & PAK1 & NM_001128620 \\
\hline $\mathrm{ABCC} 2$ & NM_000392 & EZH2 & NM_001203248 & PAK3 & NM_001128173 \\
\hline $\mathrm{ABCC} 4$ & NM_001105515 & FAM46C & NM_017709 & PALB2 & NM_024675 \\
\hline ABCC6 & NM_001079528 & FANCA & NM_001018112 & PARP1 & NM_001618 \\
\hline $\mathrm{ABCG} 2$ & NM_004827 & FANCC & NM_001243744 & PARP2 & NM_001042618 \\
\hline ABL1 & NM_005157 & FANCD2 & NM_033084 & PAX5 & NM_001280551 \\
\hline ACVR1B & NM_020327 & FANCE & NM_021922 & PBRM1 & NM_018313 \\
\hline AKT1 & NM_005163 & FANCF & NM_022725 & PDCD1 & NM_005018 \\
\hline AKT2 & NM_001243027 & FANCG & NM_004629 & PDGFRA & NM_006206 \\
\hline AKT3 & NM_005465 & FANCL & NM_001114636 & PDGFRB & NM_002609 \\
\hline ALK & NM_004304 & FBXW7 & NM_001257069 & PDK1 & NM_002610 \\
\hline AMER1 & NM_152424 & FCGR3A & NM_001127595 & PHF6 & NM_032335 \\
\hline APC & NM_000038 & FGF10 & NM_004465 & PHKA2 & NM_000292 \\
\hline AR & NM_001011645 & FGF14 & NM_004115 & PIGF & NM_002643 \\
\hline ARAF & NM_001256197 & FGF19 & NM_005117 & PIK3CA & NM_006218 \\
\hline ARFRP1 & NM_001267546 & FGF23 & NM_020638 & PIK3CB & NM_001256045 \\
\hline ARID1A & NM_139135 & FGF3 & NM_005247 & PIK3CG & NM_002649 \\
\hline ARID1B & NM_020732 & FGF4 & NM_002007 & PIK3R1 & NM_001242466 \\
\hline ARID2 & NM_152641 & FGF6 & NM_020996 & PIK3R2 & NM_005027 \\
\hline ASXL1 & NM_001164603 & FGFR1 & NM_001174064 & PLK1 & NM_005030 \\
\hline ATIC & NM_004044 & FGFR2 & NM_001144919 & PPARD & NM_177435 \\
\hline ATM & NM_000051 & FGFR3 & NM_000142 & PPP1R13L & NM_001142502 \\
\hline ATP7A & NM_000052 & FGFR4 & NM_022963 & PPP2R1A & NM_014225 \\
\hline ATR & NM_001184 & FGR & NM_001042729 & PRDM1 & NM_182907 \\
\hline ATRX & NM_000489 & FKBP1A & NM_054014 & PRDX4 & NM_006406 \\
\hline AURKA & NM_198435 & FLT1 & NM_001160031 & PRKAA1 & NM_206907 \\
\hline AURKB & NM_001256834 & FLT3 & NM_004119 & PRKAR1A & NM_002734 \\
\hline AXIN1 & NM_003502 & FLT4 & NM_002020 & PRKCA & NM_002737 \\
\hline AXL & NM_001278599 & FOXL2 & NM_023067 & PRKCB & NM_002738 \\
\hline B2M & NM_004048 & FRK & NM_002031 & PRKCE & NM_005400 \\
\hline BAIAP3 & NM_001199096 & FUBP1 & NM_003902 & PRKCG & NM_002739 \\
\hline BAP1 & NM_004656 & FYN & NM_153048 & PRKDC & NM_006904 \\
\hline BARD1 & NM_000465 & FZD7 & NM_003507 & PRRT2 & NM_001256443 \\
\hline BCL2 & NM_000657 & GALNT14 & NM_001253827 & PTCH1 & NM_001083607 \\
\hline BCL2L2 & NM_001199839 & GATA1 & NM_002049 & PTEN & NM_000314 \\
\hline BCL6 & NM_001706 & GATA2 & NM_001145662 & PTK2 & NM_001199649 \\
\hline BCOR & NM_017745 & GATA3 & NM_002051 & PTK6 & NM_001256358 \\
\hline BCORL1 & NM_021946 & GCK & NM_033508 & PTPN11 & NM_080601 \\
\hline BCR & NM_004327 & GID4 & NM_024052 & PTPRD & NM_130391 \\
\hline BIRC5 & NM_001168 & GINS2 & NM_016095 & RAC2 & NM_002872 \\
\hline BLK & NM_001715 & GNA11 & NM_002067 & RAD50 & NM_005732 \\
\hline BLM & NM_000057 & GNA13 & NM_001282425 & RAD51 & NM_001164270 \\
\hline BRAF & NM_004333 & GNAQ & NM_002072 & RAF1 & NM_002880 \\
\hline BRCA1 & NM_007297 & GNAS & NM_016592 & RARA & NM_001024809 \\
\hline BRCA2 & NM_000059 & GPC3 & NM_001164619 & $\mathrm{RB} 1$ & NM_000321 \\
\hline BRIP1 & NM_032043 & GPR124 & NM_032777 & RET & NM_020630 \\
\hline BSG & NM_001728 & GRIN2A & NM_001134408 & RICTOR & NM_001285440 \\
\hline BTK & NM_000061 & GSK3B & NM_001146156 & RMDN2 & NM_001170793 \\
\hline C11orf30 & NM_020193 & GSTM1 & NM_000561 & RNF43 & NM_017763 \\
\hline C18orf56 & NM_001012716 & GSTM3 & NM_000849 & ROCK1 & NM_005406 \\
\hline
\end{tabular}


Table I. Continued.

\begin{tabular}{|c|c|c|c|c|c|}
\hline Gene symbol & Transcripts ID & Gene symbol & Transcripts ID & Gene symbol & Transcripts ID \\
\hline C8orf34 & NM_001195639 & GSTP1 & NM_000852 & ROS1 & NM_002944 \\
\hline CAMK2G & NM_001204492 & GSTT1 & NM_000853 & RPL13 & NM_033251 \\
\hline CAMKK2 & NM_172215 & H3F3A & NM_002107 & RPS6KA1 & NM_001006665 \\
\hline CARD11 & NM_032415 & HCK & NM_001172132 & RPS6KB1 & NM_001272044 \\
\hline CASP8 & NM_033356 & HGF & NM_001010934 & RPTOR & NM_001163034 \\
\hline CBFB & NM_001755 & HIF1AN & NM_017902 & RRM1 & NM_001033 \\
\hline CBL & NM_005188 & HIST1H3B & NM_003537 & RUNX1 & NM_001122607 \\
\hline CBR1 & NM_001757 & HNF1A & NM_000545 & SDHA & NM_004168 \\
\hline CBR3 & NM_001236 & HRAS & NM_005343 & SDHAF1 & NM_001042631 \\
\hline CCND1 & NM_053056 & HSP90AA1 & NM_005348 & SDHAF2 & NM_017841 \\
\hline CCND2 & NM_001759 & IDH1 & NM_005896 & SDHB & NM_003000 \\
\hline CCND3 & NM_001136126 & IDH2 & NM_002168 & SDHC & NM_003001 \\
\hline CCNE1 & NM_001238 & IGF1 & NM_001111285 & SDHD & NM_001276506 \\
\hline CCR4 & NM_005508 & IGF1R & NM_000875 & SETD2 & NM_014159 \\
\hline CD19 & NM_001770 & IGF2 & NM_000612 & SF3B1 & NM_001005526 \\
\hline CD22 & NM_001185100 & IGF2R & NM_000876 & SGK1 & NM_005627 \\
\hline CD274 & NM_001267706 & IKBKB & NM_001556 & $\mathrm{SHH}$ & NM_000193 \\
\hline CD33 & NM_001177608 & IKBKE & NM_001193322 & SIK1 & NM_173354 \\
\hline CD38 & NM_001775 & IKZF1 & NM_001220768 & SKP2 & NM_005983 \\
\hline CD3EAP & NM_012099 & IL7R & NM_002185 & SLC10A2 & NM_000452 \\
\hline CD52 & NM_001803 & INHBA & NM_002192 & SLC15A2 & NM_001145998 \\
\hline CD74 & NM_004355 & INSR & NM_001079817 & SLC22A1 & NM_153187 \\
\hline CD79A & NM_001783 & IRF4 & NM_001195286 & SLC22A16 & NM_033125 \\
\hline CD79B & NM_000626 & IRS2 & NM_003749 & SLC22A2 & NM_003058 \\
\hline CDA & NM_001785 & ITK & NM_005546 & SLC22A6 & NM_153277 \\
\hline CDC73 & NM_024529 & JAK1 & NM_002227 & SLCO1B1 & NM_006446 \\
\hline $\mathrm{CDH} 1$ & NM_004360 & JAK2 & NM_004972 & SLCO1B3 & NM_019844 \\
\hline CDK1 & NM_001170407 & JAK3 & NM_000215 & SMAD2 & NM_001135937 \\
\hline CDK12 & NM_016507 & JUN & NM_002228 & SMAD4 & NM_005359 \\
\hline CDK2 & NM_001798 & KAT6A & NM_001099413 & SMARCA4 & NM_001128845 \\
\hline CDK4 & NM_000075 & KDM5A & NM_001042603 & SMARCB1 & NM_003073 \\
\hline CDK5 & NM_001164410 & KDM5C & NM_001146702 & SMO & NM_005631 \\
\hline CDK6 & NM_001259 & KDM6A & NM_021140 & SOCS1 & NM_003745 \\
\hline CDK7 & NM_001799 & KDR & NM_002253 & SOD2 & NM_000636 \\
\hline CDK8 & NM_001260 & KEAP1 & NM_012289 & SOX10 & NM_006941 \\
\hline CDK9 & NM_001261 & KIT & NM_000222 & SOX2 & NM_003106 \\
\hline CDKN1B & NM_004064 & KITLG & NM_003994 & SOX9 & NM_000346 \\
\hline CDKN2A & NM_001195132 & KLC3 & NM_177417 & SPEN & NM_015001 \\
\hline CDKN2B & NM_078487 & KLHL6 & NM_130446 & SPG7 & NM_199367 \\
\hline CDKN2C & NM_078626 & KMT2A & NM_001197104 & SPOP & NM_003563 \\
\hline CEBPA & NM_001285829 & KMT2B & NM_014727 & $\mathrm{SRC}$ & NM_198291 \\
\hline CHEK1 & NM_001274 & KMT2C & NM_170606 & SRD5A2 & NM_000348 \\
\hline CHEK2 & NM_001257387 & KMT2D & NM_003482 & SRMS & NM_080823 \\
\hline CHST3 & NM_004273 & KRAS & NM_033360 & STAG2 & NM_006603 \\
\hline $\mathrm{CIC}$ & NM_015125 & LCK & NM_001042771 & STAT1 & NM_139266 \\
\hline COMT & NM_007310 & LIMK1 & NM_001204426 & STAT2 & NM_005419 \\
\hline CREBBP & NM_004380 & LMO1 & NM_002315 & STAT3 & NM_003150 \\
\hline CRKL & NM_005207 & LRP1B & NM_018557 & STAT4 & NM_003151 \\
\hline CRLF2 & NM_022148 & LRP2 & NM_004525 & STAT5A & NM_003152 \\
\hline CSF1R & NM_005211 & LYN & NM_002350 & STAT5B & NM_012448 \\
\hline CSK & NM_001127190 & MAP2K1 & NM_002755 & STAT6 & NM_001178080 \\
\hline
\end{tabular}


Table I. Continued.

\begin{tabular}{|c|c|c|c|c|c|}
\hline Gene symbol & Transcripts ID & Gene symbol & Transcripts ID & Gene symbol & Transcripts ID \\
\hline CSNK1A1 & NM_001271742 & MAP2K2 & NM_030662 & STEAP1 & NM_012449 \\
\hline CTCF & NM_001191022 & MAP2K4 & NM_003010 & STK11 & NM_000455 \\
\hline CTLA4 & NM_001037631 & MAP3K1 & NM_005921 & STK3 & NM_006281 \\
\hline CTNNA1 & NM_001903 & MAP4K4 & NM_145687 & STK4 & NM_006282 \\
\hline CTNNB1 & NM_001904 & MAP4K5 & NM_198794 & SUFU & NM_001178133 \\
\hline CYBA & NM_000101 & MAPK1 & NM_138957 & SULT1A1 & NM_177534 \\
\hline CYLD & NM_001042412 & MAPK10 & NM_138981 & SULT1A2 & NM_001054 \\
\hline CYP19A1 & NM_000103 & MAPK14 & NM_139013 & SULT1C4 & NM_006588 \\
\hline CYP1A1 & NM_000499 & MAPK8 & NM_002750 & SYK & NM_001174167 \\
\hline CYP1A2 & NM_000761 & MAPK9 & NM_001135044 & TCF7L1 & NM_031283 \\
\hline CYP1B1 & NM_000104 & MAPKAPK2 & NM_004759 & TCF7L2 & NM_001198525 \\
\hline CYP2A6 & NM_000762 & MARK1 & NM_001286129 & TEK & NM_000459 \\
\hline CYP2B6 & NM_000767 & MCL1 & NM_001197320 & TET2 & NM_017628 \\
\hline CYP2C19 & NM_000769 & MDM2 & NM_001278462 & TGFBR1 & NM_004612 \\
\hline CYP2C8 & NM_001198853 & MDM4 & NM_001278516 & TGFBR2 & NM_003242 \\
\hline CYP2C9 & NM_000771 & MED12 & NM_005120 & TK1 & NM_003258 \\
\hline CYP2D6 & NM_001025161 & MEF2B & NM_001145785 & TMPRSS2 & NM_005656 \\
\hline CYP2E1 & NM_000773 & MEN1 & NM_130803 & TNF & NM_000594 \\
\hline CYP3A4 & NM_001202855 & MERTK & NM_006343 & TNFAIP3 & NM_006290 \\
\hline CYP3A5 & NM_001190484 & MET & NM_001127500 & TNFRSF10A & NM_003844 \\
\hline CYP4B1 & NM_000779 & MITF & NM_001184968 & TNFRSF10B & NM_003842 \\
\hline DAXX & NM_001254717 & MKNK2 & NM_199054 & TNFRSF14 & NM_003820 \\
\hline DDR1 & NM_001202523 & MLH1 & NM_001167617 & TNFRSF8 & NM_001243 \\
\hline DDR2 & NM_001014796 & MPL & NM_005373 & TNFSF11 & NM_003701 \\
\hline DNMT1 & NM_001130823 & MRE11A & NM_005590 & TNFSF13B & NM_001145645 \\
\hline DNMT3A & NM_153759 & MS4A1 & NM_152866 & TNK2 & NM_005781 \\
\hline DOT1L & NM_032482 & MSH2 & NM_000251 & TOP1 & NM_003286 \\
\hline DPYD & NM_001160301 & MSH6 & NM_001281494 & TP53 & NM_001276698 \\
\hline DSCAM & NM_001389 & MST1R & NM_001244937 & TPMT & NM_000367 \\
\hline E2F1 & NM_005225 & MTDH & NM_178812 & TPX2 & NM_012112 \\
\hline EGF & NM_001178131 & MTHFR & NM_005957 & TSC1 & NM_001162426 \\
\hline EGFL7 & NM_201446 & MTOR & NM_004958 & TSC2 & NM_000548 \\
\hline EGFR & NM_201283 & MTRR & NM_002454 & TSHR & NM_001018036 \\
\hline EGR1 & NM_001964 & MUTYH & NM_001048174 & TYMS & NM_001071 \\
\hline EMC8 & NM_001142288 & MYC & NM_002467 & TYRO3 & NM_006293 \\
\hline EML4 & NM_019063 & MYCL & NM_005376 & U2AF1 & NM_001025204 \\
\hline ENOSF1 & NM_001126123 & MYCN & NM_005378 & UBE2I & NM_194259 \\
\hline EP300 & NM_001429 & MYD88 & NM_001172566 & UGT1A1 & NM_000463 \\
\hline EPHA1 & NM_005232 & NAT1 & NM_001160174 & UGT1A9 & NM_021027 \\
\hline EPHA2 & NM_004431 & NAT2 & NM_000015 & UGT2B15 & NM_001076 \\
\hline EPHA3 & NM_182644 & NCAM1 & NM_001076682 & UGT2B17 & NM_001077 \\
\hline EPHA4 & NM_004438 & $\mathrm{NCF} 4$ & NM_013416 & UGT2B7 & NM_001074 \\
\hline EPHA5 & NM_001281767 & NCOA3 & NM_001174088 & UMPS & NM_000373 \\
\hline EPHA7 & NM_004440 & NCOR1 & NM_001190438 & VEGFA & NM_001171627 \\
\hline EPHA8 & NM_001006943 & NEK11 & NM_145910 & VEGFB & NM_003377 \\
\hline EPHB1 & NM_004441 & NF1 & NM_001128147 & VHL & NM_000551 \\
\hline EPHB2 & NM_004442 & NF2 & NM_181830 & WEE1 & NM_001143976 \\
\hline EPHB3 & NM_004443 & NFE2L2 & NM_001145413 & WISP3 & NM_198239 \\
\hline EPHX1 & NM_000120 & NFKBIA & NM_020529 & WNK3 & NM_020922 \\
\hline ERBB2 & NM_004448 & NKX2-1 & NM_003317 & WT1 & NM_001198552 \\
\hline ERBB3 & NM_001005915 & NOS3 & NM_001160111 & XPC & NM_001145769 \\
\hline
\end{tabular}


Table I. Continued.

\begin{tabular}{llllll}
\hline Gene symbol & Transcripts ID & Gene symbol & Transcripts ID & Gene symbol & Transcripts ID \\
\hline ERBB4 & NM_005235 & NOTCH1 & NM_017617 & XPO1 \\
ERCC1 & NM_202001 & NOTCH2 & NM_001200001 & XRCC1 & NM_003400 \\
ERCC2 & NM_001130867 & NPM1 & NM_001037738 & XRCC4 & NM_022406 \\
ERG & NM_001136155 & NQO1 & NM_000903 & YES1 & NM_005433 \\
ESR1 & NM_000125 & NRAS & NM_002524 & ZAP70 & NM_207519 \\
ETV1 & NM_001163151 & NTRK1 & NM_002529 & ZC3HAV1 & NM_024625 \\
ETV4 & NM_001261439 & NTRK2 & NM_001007097 & ZNF217 & NM_006526 \\
ETV5 & NM_004454 & NTRK3 & NM_001007156 & ZNF703 & NM_025069 \\
\hline
\end{tabular}

Genes targeted for rearrangement detection

\begin{tabular}{|c|c|c|c|c|c|}
\hline Gene symbol & Transcripts ID & Gene symbol & Transcripts ID & Gene symbol & Transcripts ID \\
\hline ALK & NM_004304 & ETV6 & NM_001987 & MYC & NM_002467 \\
\hline BCR & NM_004327 & EWSR1 & NM_001163287 & NTRK1 & NM_002529 \\
\hline BRAF & NM_004333 & KMT2A & NM_001197104 & PDGFRA & NM_006206 \\
\hline EGFR & NM_201283 & RAF1 & NM_002880 & ROS1 & NM_002944 \\
\hline ETV1 & NM_001163151 & RARA & NM_001024809 & CRLF2 & NM_022148 \\
\hline ETV4 & NM_001261439 & RET & NM_020630 & & \\
\hline ETV5 & NM_004454 & TMPRSS2 & NM_005656 & & \\
\hline
\end{tabular}

The genes and transcripts by the Novogene Comprehensive Panel. This assay covers all exons and introns spanning recurrent fusion breakpoints in v64 of the COSMIC database.

Table II. Mix ratio for cell lines.

\begin{tabular}{lcr}
\hline Cell line & Volume & Ratio \\
\hline GM19114 & 0.04 & 1 \\
GM19108 & 0.08 & 2 \\
RL95-2 & 0.08 & 2 \\
LOVO & 0.16 & 4 \\
GM18511 & 0.16 & 4 \\
HCT-15 & 0.32 & 8 \\
GM18488 & 0.64 & 16 \\
GM18957 & 6.52 & 163 \\
Total & 8 & 200 \\
\hline
\end{tabular}

In order to get more gold-standard variants with mutant allele frequencies from 0.5 to $20 \%$, cell lines were mixed in designed proportions.

culture flasks at a concentration of $1 \times 10^{5}$ viable cells $/ \mathrm{ml}$ and incubated at $37^{\circ} \mathrm{C}$ in a humidified atmosphere containing $5 \%$ $\mathrm{CO}_{2}$.

Library preparation and sequencing. Generally, genome DNA extracted was performed using DNeasy Blood \& Tissue kit (Qiagen, Hilden, Germany). For FFPE sample special, DNA was isolated using the GeneRead DNA FFPE kit (Qiagen,
Table III. Distribution of expected mutant allele frequencies in SNV and INDEL test set.

Expected mutant

allele frequency No. of sites (SNV) No. of sites (INDEL)

\begin{tabular}{lrr}
\hline$<0.5 \%$ & 568 & 32 \\
$0.5-1 \%$ & 446 & 31 \\
$1-2 \%$ & 224 & 29 \\
$2-3 \%$ & 81 & 10 \\
$3-4 \%$ & 390 & 31 \\
$4-5 \%$ & 278 & 17 \\
$5-10 \%$ & 393 & 19 \\
$>10 \%$ & 73 & 3 \\
Total & 2453 & 172 \\
\hline
\end{tabular}

Mixed cell lines contained gold-standard variants with mutant allele frequencies ranging from 0.5 to $20 \%$. These variants were used to calculate the detection performance of our assay.

Valencia, CA, USA) following the protocol. Besides the purification of high yields of DNA from FFPE tissue sections, this kit could remove deaminated cytosine to prevent false results in sequencing (27). The ratio of absorbance at 260 and $280 \mathrm{~nm}$ is used to assess the purity of extracted DNA, and we used the Qubit ${ }^{\circledR}$ Quantitation Platform to quantitated DNA. A Covaris S220 focused-ultrasonicator (Covaris, Woburn, MA, USA) 


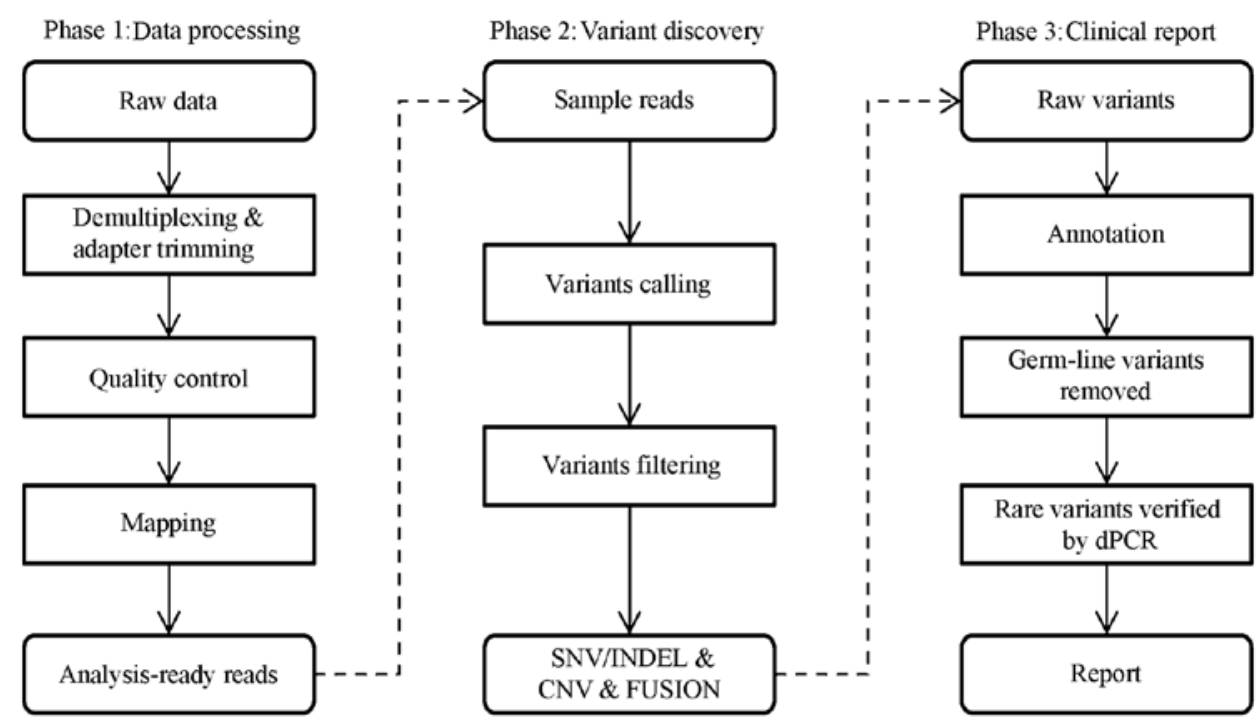

Figure 3. Framework for variation discovery. See text for a detailed description.

was used to fragment genomic DNA (500 ng) and an Agilent Bioanalyzer 2100 (Agilent Technologies) to ensure an average fragment size of 200 to 400 base pair (bp). The library preparation after fragmentation were done using instruction manual of KAPA Hyper Prep kit. The protocol included: i) repairing the DNA ends; ii) adding ' $A$ ' base to the DNA fragments; iii) ligating the paired-end adaptor; iv) purifying the sample using AMPure XP beads; and v) amplifying the adaptor-ligated library and purifying the sample using AMPure XP beads. Prepared library was hybridized using NCP custom designed baits as described in SureSelectQXT (Agilent Technologies) and the product was then amplified for 14 PCR cycles. The size range of the prepared library was assessed using Agilent 2100 Bioanalyzer and qualified using ABI StepOnePlus. The concentration of each library was quantified using qPCR NGS Library Quantification kit and Protocol was used to calculate the final pooling volume to sequencing. The products were sequenced using the Illumina HiSeq $X$ platform with pairedend sequencing runs $(2 \times 150)$ under Illumnina recommended protocols.

Data analysis. Clean data were generated by data processing steps including base calling, demultiplexing and adapter trimming. All these steps were performed using Illumina HiSeq $\mathrm{X}$ vendor software on default parameters. We further performed our in-house software for clean data quality control (QC) which included: i) removing read pairs if any one of the two reads containing base ' $\mathrm{N}$ ' $>10 \%$; ii) removing read pairs if any one of the two reads containing base with quality below Q10 $>50 \%$; iii) trimming the $3^{\prime}$ end of the read from the first base below Q20; and iv) removing reads shorter than $100 \mathrm{bp}$. Clean data after QC were mapped to the human reference genome (GRCh37) using BWA aligner v0.7.8 (28) with the default parameters. PCR duplicate read removal was done using Picard 1.119 (http://picard.sourceforge.net/index.html). According to the result, a sequence metric collection was generated including the number of total reads, percentage of reads mapped, on target reads number, average target coverage and percentage of target region with $>200 X$ and $1000 X$ coverage. Before SNV and INDEL calling, local realignment was performed using Genome Analysis Toolkit (GATK version 2.7-2-g6bda569) $(29,30)$ with default parameters and recommended 'known sites' in GATK best practice (https://software.broadinstitute. org/gatk/best-practices/). For SNV detection, we denote the reference allele and the coverage of each site as $r$ and $d$ and denote the error rate corresponding to the base calling at read $i(i=1 \ldots \mathrm{d})$ as $e_{i}$. We used a null model to explain the data in which there is no SNV at that site and all non-reference alleles to be sequencing error. The number of variant bases $(\mathrm{k})$ with $e_{i}<1 \mathrm{e}^{-3}$ (associated Phred-like quality score $q_{i}>30$ ) in each site was then given a binomial distribution. The probability under this null model was given by the following formula:

$$
\mathrm{P}(X \geq k \mid d)=1-\sum_{i=0}^{k-1} P(X=i \mid d)
$$

where $\mathrm{P}(\mathrm{X}=i \mathrm{ld})$ was the probability of observing $i$ variants in the $d$ reads of the site. Assuming the sequencing errors were independent across reads and occurred with probability $e_{0}$ $\left(e_{0}=1 \mathrm{e}^{-3} / 3\right)$ to each non-reference allele. We could obtain

$$
P(X=i \mid d)=\left(\begin{array}{l}
d \\
k
\end{array}\right) e_{0}^{k}\left(1-e_{0}\right)^{d-k}
$$

The $\mathrm{P}$-value was then given by $\mathrm{P}(\mathrm{X} \geq \mathrm{kld})$ and the cut-off $\left(\mathrm{P}\right.$-value $<1 \mathrm{e}^{-6}$ ) was established to eliminate random sequencing error. For INDEL detection, we simply kept variants supporting reads $>10$. We also employed several filters to reduce systematic errors. Empirical filters including strand bias (Fisher's exact test, $\mathrm{P}<1 \mathrm{e}^{-6}$ ), site median base quality (MBQ $>30$ ), site median mapping quality (MMQ $>30$ ), variant MAF (MAF $>0.5 \%$ ). Variants pass filters were annotated by dbSNP b146, My Cancer Genome database (https://www.mycancergenome. org) and Oncomine database v1.4.1 to get the clinical relevant information. However, cross library contamination may occur and a report would not be generated once the sample contained $>10$ variants with low-MAF (MAF $\leq 10 \%$ ) in dbSNP. In the report stage, all annotated variants with $\mathrm{MAF} \geq 5 \%$ would be reported and other cancer-related variants would be validated by $3 \mathrm{dPCR}$. The whole workflow for the data analysis is outlined in Fig. 3. The parameters and descriptions used are listed in Table IV. 
Table IV. Description of filters in data analysis.

\begin{tabular}{|c|c|}
\hline Data analysis & Description and thresholds \\
\hline Quality control & $\begin{array}{l}\text { Remove read pairs with low quality, which may lead to false positive in downstream process. Four tests } \\
\text { are used to identify such read pairs: i) read pair with one of the two reads containing base ' } N \text { ' }>10 \% \text {; } \\
\text { ii) read pairs with any one of the two reads containing base with quality below Q10 }>50 \% \text {; iii) trimming the } \\
\text { 3' end of the read from the first base below Q20; and iv) removing reads }<100 \mathrm{bp} \text {. }\end{array}$ \\
\hline Mapping & $\begin{array}{l}\text { Reads are mapped to human reference using BWA aligner v0.7.8 with BWA-MEM algorithm and relevant } \\
\text { default parameters. }\end{array}$ \\
\hline Realignment & $\begin{array}{l}\text { The GATK realignment is used to correct the misalignment due to the presence of an INDEL. This step } \\
\text { use two files 'Mills_and_1000G_gold_standard.indels.b37.sites.vcf' and '1000G_phase1.indels.b37.vcf' } \\
\text { (https://software.broadinstitute.org/gatk/best-practices/) to get these INDEL. The default parameters are } \\
\text { used to perform the realignment. }\end{array}$ \\
\hline Call SNV & $\begin{array}{l}\text { A binomial test is used to separate true positive from noises. The P-value cut-off is } 1 \mathrm{e}^{-6} \text {, and the } \\
\text { probability of sequencing error is } 1 \mathrm{e}^{-3} / 3 \text {. }\end{array}$ \\
\hline Call INDEL & A cut-off of 10 support reads is used to call INDEL. \\
\hline Hard filter & $\begin{array}{l}\text { To further remove false positives, several hard filters have been used as follows: i) Fisher's exact test for } \\
\text { strand bias, P-value }<1 \mathrm{e}^{-6} \text {. Some false positives are generated in sequencing step and have close relation- } \\
\text { ship to the front of the sequence (homopolymer or other special sequence); ii) site median base quality } \\
>30 \text {. In case of the base quality of each read could not represent the true error rate, the median base } \\
\text { quality of each site is used to evaluate such error rate; iii) site median mapping quality }>30 \text {. This filter } \\
\text { is used to avoid the misalignment of repeat sequences with small difference in human reference which are } \\
\text { easily mistaken as SNV. }\end{array}$ \\
\hline
\end{tabular}

These filters were obtained from clinical samples and covered all special cases that we had met before. Therefore, it could identify true positive variants from most noise in sequencing.

Compared with other software. To measure the effect of our approach, we compared the pooled cell-line result with GATK, a widely used software. We followed the 'GATK best practice', the 'IndelRealigner' parameter 'LOD_ Threshold_For_Cleaning' was 0.3, the 'BaseRecalibrator' was with default parameters, the SNV/INDEL calling type was 'HaplotypeCaller' with parameters 'standard_min_confidence_threshold_for_emitting' as 10 and 'standard_min_confidence_threshold_for_calling' as 30 .

Performance statistics calculation. For sensitivity estimation, variants detected in pools would be assigned as true positive (TP), or false negative (FN) if not detected. Sensitivity was calculated as TP/(TP+FN). For specificity estimation, the pool variants also detected in the pure sample were assigned as true positive (TP), or false positive (FP) if none was detected. PPV was calculated as $\mathrm{TP} /(\mathrm{TP}+\mathrm{FP})$.

Mutation detection by $d P C R$. dPCR is a method used in absolute quantification analysis of clonally amplified nucleic acids (including DNA, cDNA, methylated DNA or RNA). With dPCR, a sample is partitioned so that individual nucleic acid molecules within the sample are localized and concentrated within many separate regions. After PCR amplification, nucleic acids may be quantified by counting the regions that contain PCR end-product, positive reactions. Here, we used the QuantStudio ${ }^{\text {TM }}$ 3D Digital PCR System platform (Life Technologies) regarding SNP mutation quantitation.
For dPCR, the first step is preparing and loading samples onto QuantStudio $^{\mathrm{TM}}$ 3D Digital PCR 20K chips. Mutations were analysed by TaqMan ${ }^{\circledR}$ SNP Genotyping Assays (Life Technologies), which containing TaqMan ${ }^{\circledR}$-MGB probes and primers. We prepared $15 \mu \mathrm{l}$ reaction mixes according to the manufacturer's instructions, and loaded $14.5 \mu \mathrm{l}$ onto each chip. The Mix contains ROX ${ }^{\circledR}$ dye, which served as a passive reference. After chips were loaded, we run the Digital PCR 20K Chips with a ProFlex ${ }^{\mathrm{TM}} 2 \mathrm{x}$ Flat PCR System under the following conditions: $96^{\circ} \mathrm{C}$ for $10 \mathrm{~min}, 39$ cycles at $56^{\circ} \mathrm{C}$ for $2 \mathrm{~min}$ and at $98^{\circ} \mathrm{C}$ for $30 \mathrm{sec}$, followed by a final extension step at $56^{\circ} \mathrm{C}$ for $2 \mathrm{~min}$. After thermo-cycling, we analyzed the prepared chips using dPCR instrument.

Mutation detection by ARMS-PCR. ARMS-PCR is a realtime PCR-based test which covers the 29 EGFR hotspots from exon 18-21. The assay was performed according to the manufacturer's protocol for the ADx EGFR29 Mutation kit (Amoy Diagnostics, Co., Ltd., Xiamen, China) with the MX3000P (Stratagene, La Jolla, CA, USA) real-time PCR system. Template DNA $(0.4 \mu \mathrm{l}), 3.6 \mu \mathrm{l}$ deionized water and $16 \mu \mathrm{l}$ other reaction components was used in the RT-PCR reaction system. PCR was performed with initial denaturation at $95^{\circ} \mathrm{C}$ for $10 \mathrm{~min}$, followed by 40 cycles of amplification (at $95^{\circ} \mathrm{C}$ for $30 \mathrm{sec}$ and $61^{\circ} \mathrm{C}$ for $1 \mathrm{~min}$ ). The results were analyzed according to the criteria defined by the manufacturer's instructions. Positive results were defined as [Ct(sample) - Ct(control) $]<\mathrm{Ct}$ (cut-off). 
Table V. Summary of sequencing metrics for cell lines.

\begin{tabular}{lcccccc}
\hline Cell line & Total read pairs (M) & Total bases $(\mathrm{Mb})$ & $\begin{array}{c}\text { Mapped } \\
\text { baseNum }(\mathrm{Mb})\end{array}$ & $\begin{array}{c}\text { BaseNum } \\
\text { on target }(\mathrm{Mb})\end{array}$ & $\begin{array}{c}\text { Covered at least } \\
\text { 200X }(\%)\end{array}$ & $\begin{array}{c}\text { Median target } \\
\text { coverage (X) }\end{array}$ \\
\hline GM18511 & 151 & 22,595 & 13,567 & 7,920 & 99.60 & 3405.41 \\
GM18957 & 84 & 12,633 & 8,875 & 5,215 & 99.30 & 2242.34 \\
GM19114 & 61 & 9,130 & 7,216 & 4,438 & 99.10 & 1908.01 \\
GM19108 & 73 & 10,923 & 7,745 & 4,004 & 98.80 & 1721.50 \\
GM18488 & 82 & 12,295 & 8,810 & 4,472 & 98.90 & 1922.71 \\
RL95-2 & 83 & 12,405 & 8,893 & 4,161 & 98.80 & 1788.99 \\
HCT-15 & 88 & 13,217 & 9,254 & 4,811 & 99.00 & 2068.53 \\
LoVo & 90 & 13,444 & 9,453 & 4,950 & 98.90 & 2128.07 \\
\hline
\end{tabular}

Pure cell lines used to establish the SNV and INDEL test set.

Table VI. Summary of sequencing metrics for mixed cell lines pool.

\begin{tabular}{lcccccc}
\hline Pool name & $\begin{array}{c}\text { Total read } \\
\text { pairs }(\mathrm{M})\end{array}$ & $\begin{array}{c}\text { Total } \\
\text { bases }(\mathrm{Mb})\end{array}$ & $\begin{array}{c}\text { Mapped } \\
\text { baseNum }(\mathrm{Mb})\end{array}$ & $\begin{array}{c}\text { BaseNum } \\
\text { on target }(\mathrm{Mb})\end{array}$ & $\begin{array}{c}\text { Covered at least } \\
\text { 200X }(\%)\end{array}$ & $\begin{array}{c}\text { Median target } \\
\text { coverage (X) }\end{array}$ \\
\hline 5G & 32 & 4,762 & 4,591 & 2,393 & 97.50 & 1028.96 \\
10G & 73 & 10,896 & 10,316 & 5,202 & 99.20 & 2236.63 \\
20G & 109 & 16,351 & 15,138 & 7,429 & 99.50 & 3194.21 \\
\hline
\end{tabular}

Cell line pools were used to calculate variants detection performance.

\section{Results}

Overview. NCP is a NGS-based clinical test for detection of somatic cancer related mutations. DNA was extracted from tumor tissues and FFPE samples, $500 \mathrm{ng}$ of which was fragmented, captured using custom-designed hybridization-based biotinylated cRNA reagents and amplified via limited-cycle PCR to enrich 7,011 exons and 94 introns of 483 cancer related genes (totaling 2.3 million sites). We used clinical samples to generate the bioinformatics pipeline for data analysis (Table IV) and cell lines to validate the whole work flow. For the 8 single cell lines, using the Illumina HiSeq X platform, achieving an average of $13,330 \mathrm{Mb}(\mathrm{SD}=3,995 \mathrm{Mb})$ total bases with $38.09 \%$ on-target $(\mathrm{SD}=4.78 \%)$, target regions were sequenced to 2148X $(\mathrm{SD}=537 \mathrm{X})$ median coverage across targeted bases, with $99.05 \%$ ( $\mathrm{SD}=0.28 \%$ ) of targeted bases covered by at least 200 reads (Table V). The 2453 SNV and 172 INDEL detected in single cell line consistent with database would be used for assessment of SNV/INDEL detection. Pools of mixed cell lines were used to get the relationship between median coverage and performance, which achieved total bases of 4,762, 10,896 and $16,351 \mathrm{Mb}$, the median coverage of 1,029X, 2,237X and 3,194X (Table VI). Due to the high sensitivity NGS benefit from high coverage, the hotspot mutations with MAF $<5 \%$ detected by this assay in $35 \mathrm{FFPE}$ samples were confirmed by dPCR. All samples used in this test are summarized in Table VII. Finally, 33 hotspot mutations detected by NGS in FFPE samples with a MAF from 2 to $63 \%$ in NGS were tested by ARMS-PCR.
SNV detection performance. SNV detection was performed using a Binomial methodology allowing the detection of low MAF somatic mutations across the $2.3 \mathrm{Mb}$ assayed with high sensitivity. For the mixed cell line pools, overall SNV detection performance was high, the results of different depth are shown in Table VIII, for an average depth of 2237, $100 \%$ (95\% CI, 95.1-100\%) of SNV at MAF $>10 \%$ were successfully detected, as well as $99 \%$ (95\% CI, 98.6-100\%) of SNV at MAF 5-10\%. The detection of SNV with MAF between $0.5-5 \%$ performance was 92.2\% (95\% CI, 90.7-93.5\%) (Fig. 4A and C and Table VIIIA). In addition, high sensitivity was accompanied with good PPV (the fraction of SNV calls in the pools can also be detected in any of the individual cell lines; Table VIIIB) 99.2\% (95\% CI, 99-99.4\%). The false positives may be due to variants with such a low MAF $(<5 \%)$ no difference with sequencing noise could hardly be identified. A dPCR confirmation for cancer-related SNV with MAF $<5 \%$ reported by NGS is necessary before reporting.

INDEL detection performance. For INDEL detection, we simply discarded the variants supporting less than 10 reads. The results of different depth are shown in Table IX, for an average depth of 2237, $100 \%$ (95\% CI, 29.2-100\%) of INDEL at MAF $>10 \%$ were successfully detected, as well as $94.7 \%$ of INDEL (95\% CI, 74-99.9\%) with MAF between 5-10\%. Low MAF sites detected performance was $91.5 \%$ (95\% CI, $85-100 \%$ ), the performance of variants with MAF $<0.5 \%$ was also calculated (Fig. 4B and D and Table IXA). Few falsepositive calls were observed, with a PPV of $98.2 \%$ (95\% CI, 97.2-98.9\%) (Table IXB). Like SNV detection, due to the false 
Table VII. Overview of study objectives and strategy.

\begin{tabular}{llcccc} 
Objective & \multicolumn{1}{c}{ Sample set } & \#Samples & $\begin{array}{c}\text { Sample } \\
\text { type }\end{array}$ & $\begin{array}{c}\text { DNA } \\
\text { input (ng) }\end{array}$ & $\begin{array}{c}\text { Sequencing } \\
\text { platform }\end{array}$ \\
\hline $\begin{array}{l}\text { Individual cell line SNP consistent with } \\
\text { database gold standard }\end{array}$ & $\begin{array}{l}\text { Cell lines with known } \\
\text { SNPs and INDELs }\end{array}$ & 8 & Cell line & 500 & Hiseq-X \\
$\begin{array}{l}\text { Cell line pools to validate } \\
\text { SNP/INDEl performance }\end{array}$ & $\begin{array}{l}\text { Cell lines at specific } \\
\text { ratio in 3 pools }\end{array}$ & 3 & Cell line & 500 \\
$\begin{array}{l}\text { Confirm specificity (MAF }<5 \%) \\
\text { Confirm specificity (all MAF) }\end{array}$ & $\begin{array}{l}\text { Clinical FFPE samples } \\
\text { CFP samples }\end{array}$ & 35 & FFPE & $300-500$ \\
\hline
\end{tabular}

The first phase of this study was focused on analytical performance validation. It was performed by using 8 cell lines with known allele frequencies for analytical detection analysis. The second phase focused on clinical FFPE samples. Sixty-eight clinical samples were used to compare variants detection in NGS with other approaches. MAF, mutant allele frequency.

Table VIII. Summary of SNV detection performance (sensitivity, ppv).

A, Summary of SNV detection performance (sensitivity)

\begin{tabular}{|c|c|c|c|c|c|c|c|c|c|c|c|c|}
\hline \multirow{2}{*}{$\begin{array}{l}\text { Average } \\
\text { coverage }\end{array}$} & \multicolumn{3}{|c|}{$\begin{array}{c}\text { MAF }<0.5 \% \\
n=568\end{array}$} & \multicolumn{3}{|c|}{$\begin{array}{c}\text { MAF } 0.5-5 \% \\
n=1419\end{array}$} & \multicolumn{3}{|c|}{$\begin{array}{c}\text { MAF } 5-10 \% \\
n=393\end{array}$} & \multicolumn{3}{|c|}{$\begin{array}{c}\text { MAF }>10 \% \\
n=73\end{array}$} \\
\hline & FN & SEN (\%) & CI (\%) & $\mathrm{FN}$ & SEN (\%) & $\mathrm{CI}(\%)$ & $\mathrm{FN}$ & SEN (\%) & $\mathrm{CI}(\%)$ & $\mathrm{FN}$ & SEN (\%) & $\mathrm{CI}(\%)$ \\
\hline 3194 & 471 & 17.1 & $14.1-20.4$ & 86 & 93.9 & $92.6-95.1$ & 0 & 100 & $99.1-100$ & 0 & 100 & $95.1-100$ \\
\hline 2237 & 446 & 21.5 & $18.2-25.1$ & 111 & 92.2 & $90.7-93.5$ & 1 & 99.8 & 98.6-100 & 0 & 100 & $95.1-100$ \\
\hline 1029 & 446 & 21.5 & $18.2-25.1$ & 164 & 88.4 & $86.7-90.1$ & 2 & 99.5 & $98.2-100$ & 0 & 100 & $95.1-100$ \\
\hline
\end{tabular}

B, Summary of SNV detection performance (specificity)

\begin{tabular}{|c|c|c|c|c|c|}
\hline \multirow[b]{2}{*}{ Average coverage } & \multirow[b]{2}{*}{$\mathrm{TP}$} & \multicolumn{2}{|c|}{ FP } & \multicolumn{2}{|c|}{ PPV } \\
\hline & & $\mathrm{MAF} \geq 5 \%$ & MAF $<5 \%$ & Mean $(\%)$ & CI $(\%)$ \\
\hline 3194 & 5720 & 0 & 84 & 98.5 & $98.2-98.8$ \\
\hline 2237 & 5619 & 0 & 43 & 99.2 & $99.0-99.4$ \\
\hline 1029 & 4661 & 0 & 4 & 99.9 & $99.8-100$ \\
\hline
\end{tabular}

The SNV detection performance of our pipeline in analytical validation. False negatives were germ line SNPs in constituent cell lines that were not detected in mixed cell line data. False positives were SNV calls in pooled samples absent from pure cell lines. MAF, mutation allele frequency; FN, false negative; SEN, sensitivity; CI, confidence interval (calculated as the exact 95\% binomial confidence interval).

positive under $10 \%$, a dPCR confirmation of these cancerrelated INDEL with MAF $<10 \%$ before reporting is needed.

Comparison with other bioinformatics approaches. We evaluated the performance of our bioinformatics pipeline with the cell line models above, focusing on two key steps of our approach. First, we applied statistical models that allow for the identification of a mutation at low MAF from random errors in Illumina sequencing. Second, we used priori knowledge to identify systematic errors always accompanied with specific characteristics, such as strand bias and low base/ mapping quality. To measure the effect of our approach, we compared the pooled cell-line result with GATK - widely used software. The GATK detection sensitivity of SNV with MAF $>10 \%$ was $64.38 \%$ (95\% CI, 52.3-75.3\%), and SNV with $5 \%<\mathrm{MAF}<10 \%$ was under $10 \%$ but the PPV was $100 \%$ (95\% CI, 99.7-100\%). The sensitivity of INDEL with MAF $>10 \%$ was $67 \%$ (95\% CI, 9.4-99.2\%), and a high PPV $100 \%$ (95\% CI, 99-100\%) (Tables X and XI), possibly because this widely used tool is designed for whole-genome or wholeexon sequencing data with relatively low depth and variants with high allele frequency, which underline that appropriate 
Table IX. Summary of INDEL performance (sensitivity, ppv).

A, Summary of small insert and deletion detection performance (sensitivity)

\begin{tabular}{|c|c|c|c|c|c|c|c|c|c|c|c|c|}
\hline \multirow{2}{*}{$\begin{array}{l}\text { Average } \\
\text { coverage }\end{array}$} & \multicolumn{3}{|c|}{$\begin{array}{c}\text { MAF }<0.5 \% \\
n=32\end{array}$} & \multicolumn{3}{|c|}{$\begin{array}{c}\text { MAF } 0.5-5 \% \\
n=118\end{array}$} & \multicolumn{3}{|c|}{$\begin{array}{c}\text { MAF 5-10\% } \\
n=19\end{array}$} & \multicolumn{3}{|c|}{$\begin{array}{c}\text { MAF }>10 \% \\
n=3\end{array}$} \\
\hline & FN & $\operatorname{SEN}(\%)$ & CI $(\%)$ & $\mathrm{FN}$ & $\operatorname{SEN}(\%)$ & CI (\%) & $\mathrm{FN}$ & SEN (\%) & CI (\%) & FN & SEN (\%) & CI (\%) \\
\hline 3194 & 24 & 25.0 & $11.5-43.4$ & 9 & 92.4 & $86-96.5$ & 0 & 100 & $82.4-100$ & 0 & 100 & $29.2-100$ \\
\hline 2237 & 26 & 18.8 & $7.2-36.4$ & 10 & 91.5 & $85-95.9$ & 1 & 94.7 & 74-99.9 & 0 & 100 & $29.2-100$ \\
\hline 1029 & 25 & 21.9 & $9.3-40$ & 15 & 87.3 & $79.9-92.7$ & 2 & 89.5 & $66.9-98.7$ & 0 & 100 & $29.2-100$ \\
\hline
\end{tabular}

B, Summary of small insert and deletion detection performance (specificity)

\begin{tabular}{lcccccr}
\hline & & \multicolumn{2}{c}{ FP } & & \multicolumn{2}{c}{ PPV } \\
\cline { 3 - 4 } Average coverage & TP & MAF $>10 \%$ & MAF < 10\% & & Mean (\%) & CI (\%) \\
\hline 3194 & 1119 & 0 & 24 & 97.9 & $96.8-98.6$ \\
2237 & 1050 & 0 & 19 & 98.2 & $97.2-98.9$ \\
1029 & 794 & 0 & 13 & 98.4 & $97.2-99.1$ \\
\hline
\end{tabular}

The INDEL detection performance of our pipeline. INDEL calls in pooled samples had the same base composition and position $( \pm 25$ bp) which were considered to be true positives. False positives were INDEL calls in pooled samples that were absent from pure cell lines. MAF, mutation allele frequency; FN, false negative; SEN, sensitivity; CI, confidence interval (calculated as the exact $95 \%$ binomial confidence interval).
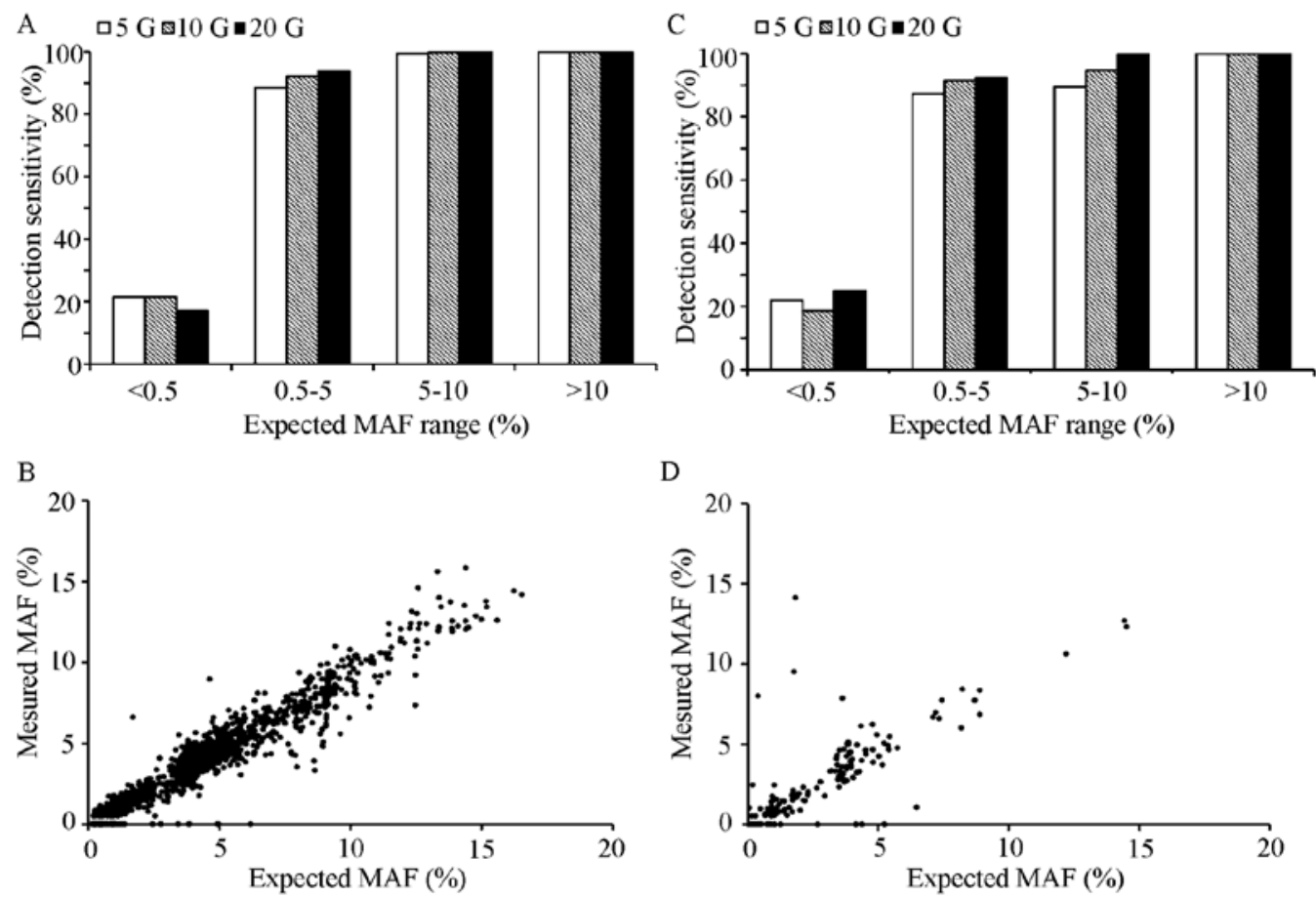

Figure 4. SNV and INDEL detection performance. (A) SNV detection sensitivity for different data size as a function of variants except MAF. (B) SNV allele frequencies measured in pooled samples (y-axis) match the frequencies expected based on the genotypes and mixing ratios of constituent cell lines (x-axis). (C) INDEL detection sensitivity for different data size as a function of variants except MAF. (D) INDEL allele frequencies measured in pooled samples (y-axis) match the frequencies expected based on the genotypes and mixing ratios of constituent cell lines (x-axis).

filters for ultra-deep sequencing data analysis were critical. Actually, compared with slight performance upgrades under increased coverage depth, the effect of appropriate filters was remarkable in this test. 
Table X. Summary of SNV detection performance by GATK (sensitivity, ppv).

A, Summary of SNV detection performance by GATK (sensitivity)

\begin{tabular}{|c|c|c|c|c|c|c|c|c|c|c|c|c|}
\hline \multirow{2}{*}{$\begin{array}{l}\text { Average } \\
\text { coverage }\end{array}$} & \multicolumn{3}{|c|}{$\begin{array}{c}\text { MAF }<0.5 \% \\
n=568\end{array}$} & \multicolumn{3}{|c|}{$\begin{array}{c}\text { MAF } 0.5-5 \% \\
n=1419\end{array}$} & \multicolumn{3}{|c|}{$\begin{array}{c}\text { MAF } 5-10 \% \\
n=393\end{array}$} & \multicolumn{3}{|c|}{$\begin{array}{c}\text { MAF }>10 \% \\
n=73\end{array}$} \\
\hline & $\mathrm{FN}$ & $\operatorname{SEN}(\%)$ & CI $(\%)$ & FN & $\operatorname{SEN}(\%)$ & CI (\%) & FN & SEN (\%) & $\mathrm{CI}(\%)$ & FN & SEN (\%) & CI (\%) \\
\hline 3194 & 567 & 0.18 & $0-1$ & 1417 & 0.14 & $0-0.5$ & 374 & 4.83 & $2.9-7.4$ & 18 & 75.34 & $63.9-84.7$ \\
\hline 2237 & 568 & 0.00 & $0-0.6$ & 1417 & 0.14 & $0-0.5$ & 370 & 5.85 & $3.7-8.7$ & 26 & 64.38 & $52.3-75.3$ \\
\hline 1029 & 567 & 0.18 & $0-1$ & 1416 & 0.21 & $0-0.6$ & 375 & 4.58 & $2.7-7.1$ & 25 & 65.75 & $53.7-76.5$ \\
\hline
\end{tabular}

B, Summary of SNV detection performance by GATK (specificity)

\begin{tabular}{|c|c|c|c|c|c|}
\hline \multirow[b]{2}{*}{ Average coverage } & \multirow[b]{2}{*}{$\mathrm{TP}$} & \multicolumn{2}{|c|}{ FP } & \multicolumn{2}{|c|}{ PPV } \\
\hline & & $\mathrm{MAF} \geq 5 \%$ & MAF $<5 \%$ & Mean $(\%)$ & CI (\%) \\
\hline 3194 & 2212 & 1 & 0 & 100.0 & $99.7-100$ \\
\hline 2237 & 2213 & 1 & 0 & 100.0 & $99.7-100$ \\
\hline 1029 & 2188 & 0 & 0 & 100.0 & $99.8-100$ \\
\hline
\end{tabular}

The SNV detection performance of GATK pipeline in mixed cell lines. False negatives were germ line SNPs in constituent cell lines that were not detected in mixed cell line data. False positives were SNV calls in pooled samples that were absent from pure cell lines. CI, confidence intervals (calculated as the exact 95\% binomial confidence interval); MAF, mutation allele frequency. FN, false negative; SEN, sensitivity.

Table XI. Summary of INDEL detection performance by GATK (sensitivity, ppv).

A, Summary of INDEL detection performance by GATK (sensitivity)

\begin{tabular}{|c|c|c|c|c|c|c|c|c|c|c|c|c|}
\hline \multirow{2}{*}{$\begin{array}{l}\text { Average } \\
\text { coverage }\end{array}$} & \multicolumn{3}{|c|}{$\begin{array}{c}\text { MAF }<0.5 \% \\
n=32\end{array}$} & \multicolumn{3}{|c|}{$\begin{array}{c}\text { MAF } 0.5-5 \% \\
n=118\end{array}$} & \multicolumn{3}{|c|}{$\begin{array}{c}\text { MAF } 5-10 \% \\
n=19\end{array}$} & \multicolumn{3}{|c|}{$\begin{array}{c}\text { MAF }>10 \% \\
n=3\end{array}$} \\
\hline & FN & $\operatorname{SEN}(\%)$ & $\mathrm{CI}(\%)$ & $\mathrm{FN}$ & SEN (\%) & $\mathrm{CI}(\%)$ & FN & SEN (\%) & CI (\%) & $\mathrm{FN}$ & SEN (\%) & CI $(\%)$ \\
\hline 3194 & 31 & 3.13 & $0.1-16.2$ & 116 & 1.69 & $0.2-6$ & 16 & 15.79 & $3.4-39.6$ & 0 & 100.00 & $29.2-100$ \\
\hline 2237 & 31 & 3.13 & $0.1-16.2$ & 116 & 1.69 & $0.2-6$ & 18 & 5.26 & $0.1-26$ & 1 & 67 & $9.4-99.2$ \\
\hline 1029 & 31 & 3.13 & $0.1-16.2$ & 116 & 1.69 & $0.2-6$ & 17 & 10.53 & $1.3-33.1$ & 0 & 100.00 & $29.2-100$ \\
\hline
\end{tabular}

B, Summary of INDEL detection performance by GATK (specificity)

\begin{tabular}{|c|c|c|c|c|c|}
\hline \multirow[b]{2}{*}{ Average coverage } & \multirow[b]{2}{*}{$\mathrm{TP}$} & \multicolumn{2}{|c|}{ FP } & \multicolumn{2}{|c|}{ PPV } \\
\hline & & MAF $>10 \%$ & MAF $<10 \%$ & Mean (\%) & $\mathrm{CI}(\%)$ \\
\hline 3194 & 385 & 0 & 0 & 100.0 & $99-100$ \\
\hline 2237 & 386 & 0 & 0 & 100.0 & $99-100$ \\
\hline 1029 & 380 & 0 & 0 & 100.0 & $99-100$ \\
\hline
\end{tabular}

The INDEL detection performance of GATK pipeline. INDEL calls in pooled samples had the same base composition and position ( \pm 25 bp) which were considered to be true positives. False positives were INDEL calls in pooled samples that were absent from pure cell lines. MAF, mutation allele frequency. FN, false negative; SEN, sensitivity; CI, confidence interval (calculated as the exact $95 \%$ binomial confidence interval).

Concordance between NGS and other approaches. The above studies demonstrate that the NGS-based test has the performance characteristics necessary to accurately detect SNV and INDEL. We further validated test accuracy by 


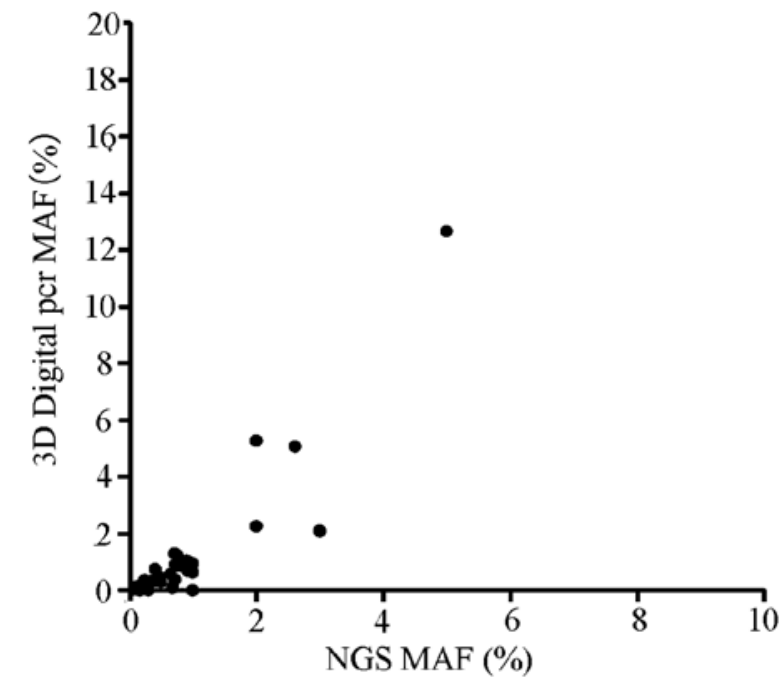

Figure 5. Correlation between NGS and dPCR. Samples with mutations detected during NGS and MAF $<5 \%$ were also tested by dPCR. The detected allele frequency correlation between 3D digital PCR and NGS is shown $\left(\mathrm{R}^{2}=0.82\right)$.

Table XII. 3D digital PCR correlation results.

\begin{tabular}{lcc}
\hline Genes and exons & NGS (no.) & $\begin{array}{c}\text { Supported by } \\
\text { 3d digital pcr (no.) }\end{array}$ \\
\hline PPIK3CA exon 9 & 1 & 1 \\
PPIK3CA exon 10 & 3 & 3 \\
PPIK3CA exon 21 & 1 & 1 \\
EGFR exon 18 & 1 & 0 \\
EGFR exon 19 & 6 & 6 \\
EGFR exon 20 & 11 & 10 \\
EGFR exon 21 & 5 & 4 \\
KRAS exon 2 & 5 & 5 \\
BRAF exon 15 & 1 & 1 \\
KRAS exon 3 & 1 & 1 \\
\hline
\end{tabular}

The concordance between NGS and 3D digital PCR for variants with mutant allele frequency under $5 \%$.

comparisons to dPCR for $35 \mathrm{FFPE}$ cancer specimens. To assess the accuracy of low MAF SNV and INDEL detection in routine clinical cancer samples, we selected 35 FFPE resection specimens (31 non-small cell lung cancer, 1 parathyroid carcinoma, 3 breast cancers) previously tested for hotspot mutations in PIK3Ca, EGFR, KRAS and BRAF by NGS, every hotspot mutations detected by NGS, but with $\mathrm{MAF}<5 \%$ would be tested by dPCR. In addition, 32 of 35 (PPV=91.43\%, 95\% CI, 76.94-98.20\%) variants have been supported to be true-positive by dPCR (Tables XII and XIII). Three variants were present at $<3 \%$ MAF in NGS that were not detected by dPCR. The detected MAF of the two technologies is shown in Fig. 5. Finally, we random selected 33 FFPE samples (NSCLC) with hotspot mutations and performed the ARMS-PCR to verify the overall PPV of our assay. As a result, all 33 mutations could be detected by ARMS-PCR and the PPV was $100 \%$ (95\% CI, 89.42-100\%; Table XIV).

\section{Discussion}

Cancer diagnostic is undergoing a rapid development (31), routine tests like FISH and IHC can only detect limited known variants, besides it fully relies on the doctor's experience. PCR-based approach, like Sanger sequencing or dPCR used by us in this study, still cannot test multiple sites in one run. Furthermore, Sanger sequencing cannot detect variants with MAF under 10\% (32) and dPCR waste too many samples, which remain problems for clinical application. The NGS-based test with increased access and decreased cost has more advantages in comprehensive detection of the cancer-related mutations (33-35). For detecting mutations with low frequency, NGS-based test with high sensitivity is needed. However, high sensitivity always comes with false-positives, which may lead to suboptimal treatment. Finally, some other factors, like DNA damage and contamination in clinical samples $(36,37)$, make it critical to generate a complex validation of NGS assay.

In the present study, we developed and validated the NGS-based assay, using germ line mutations in 1000 genome cell lines and certain somatic INDEL in cosmic database to simulate the tumor heterogeneity or impurity in clinical samples. We mixed these samples to measure the analytic sensitivity and PPV of NCP assay at low MAF and used 3 pools to obtain the correlation between median coverage and variants detection performance. The performance of our test was high for variants with MAF $>5 \%$. In cell line model with 2236X median coverage, sensitivity was $99.8 \%$ for SNP, $94.7 \%$ for INDEL with a PPV of 99 and $98 \%$. The $0.5 \%<\mathrm{MAF}<5 \%$ variant sensitivity was $92.2 \%$ for SNV and $91.5 \%$ for INDEL which was not desirable. Because of the complexity of 483 genes, it was difficult to ensure such low MAF variant detection sensitivity. On the other hand, we confirmed the low MAF detection by dPCR which could identify rare mutations specifically. We also compared our bioinformatics pipeline with common pipeline $\operatorname{GATK}(29,30)$, which is widely used in genotype analysis. The overall PPV was high at the expense of sensitivity, which may be due to these approaches being developed to call germ line variants. The results highlighted that appropriate filtering approach is critical for low MAF variant detection. Actually, the filters were more important than the increase of coverage depth as showed in the different coverage tests. For specificity analysis, each called variant was classified as a false positive if a matching alteration was not detected in the pure sample. However, this approach could not recognize the false positive generated by systematic errors. Given the high sensitivity of this technology, high-throughput clinical trials are required to confirm its reliability for the molecular diagnosis of cancer (38). Therefore, 35 patient specimens previously tested by NCP assay and having low MAF $<5 \%$ variants were used to test in parallel by dPCR. The correlation coefficient of NGS and APCR was low (0.78) and 32 of 35 (91.43\%) NGS detected variants could be confirmed by dPCR. The discordance was possibly due to the heterogeneity in tumor specimens or false positive in NGS, the dPCR verification is needed for such low MAF variants before reporting. Like low 
Table XIII. Summary of concordance between NGS and 3D Digital PCR.

\begin{tabular}{|c|c|c|c|c|c|}
\hline Sample id & Mutation & NGS $(\%)$ & $\mathrm{dPCR}(\%)$ & Cancer type & Stage \\
\hline d001 & EGFR:exon19:c.2235_2249del:p.746_750del & 5.00 & 12.64 & NSCLC & - \\
\hline d002 & PIK3CA:exon21:c.A3140G:p.H1047R & 3.00 & 2.09 & Breast cancer & - \\
\hline d003 & KRAS:exon2:c.G35A:p.G12D & 2.61 & 5.07 & NSCLC & 4 \\
\hline d004 & EGFR:exon19:c.2235_2249del:p.746_750del & 2.00 & 5.26 & NSCLC & 4 \\
\hline d005 & BRAF:p.V600Ec.1799T>A & 2.00 & 2.25 & NSCLC & 4 \\
\hline d006 & EGFR:exon21:c.T2573G:p.L858R & 1.00 & 0.00 & NSCLC & 4 \\
\hline d007 & EGFR:exon20:c.C2369T:p.T790M & 1.00 & 0.62 & NSCLC & 4 \\
\hline d008 & KRAS:exon2:c.G35A:p.G12D & 1.00 & 0.93 & Parathyroid carcinoma & 4 \\
\hline d009 & PIK3CA:exon9:c.1633G>A:p.E545K & 0.92 & 0.68 & NSCLC & - \\
\hline $\mathrm{d} 010$ & EGFR:exon20:c.C2369T:p.T790M & 0.90 & 1.05 & NSCLC & 4 \\
\hline d011 & EGFR:exon19:c.2236_2250del:p.746_750del & 0.79 & 0.84 & NSCLC & 3 \\
\hline $\mathrm{d} 012$ & KRAS:exon2:c.G35A:p.G12D & 0.77 & 1.20 & NSCLC & 4 \\
\hline d013 & EGFR:exon20:c.C2369T:p.T790M & 0.73 & 0.90 & NSCLC & 2 \\
\hline d014 & $\begin{array}{l}\text { EGFR:exon19:c.2235_2249delGGAATTAAGAGAAGC: } \\
\text { p.E746_A750del }\end{array}$ & 0.71 & 1.29 & NSCLC & 4 \\
\hline d015 & EGFR:exon21:c.T2573G:p.L858R & 0.71 & 0.38 & NSCLC & 4 \\
\hline $\mathrm{d} 016$ & KRAS:p.G12C:c.34G>T & 0.68 & 0.08 & NSCLC & 4 \\
\hline d017 & PIK3CA:exon10:c.G1633A:p.E545K & 0.64 & 0.57 & NSCLC & 4 \\
\hline $\mathrm{d} 018$ & EGFR:exon21:c.2573T>G:p.L858R & 0.50 & 0.29 & NSCLC & \\
\hline d019 & KRAS:exon2:c.G37T:p.G13C & 0.47 & 0.44 & NSCLC & - \\
\hline d020 & PIK3CA:c.1633G>A:p.E545K & 0.42 & 0.37 & NSCLC & 4 \\
\hline d021 & PIK3CA:exon10:c.G1624A:p.E542K & 0.41 & 0.73 & Breast cancer & 3 \\
\hline d022 & EGFR:exon19:c.2235_2249del:p.745_750del & 0.40 & 0.32 & NSCLC & 4 \\
\hline d023 & EGFR:exon21:c.2573T>G:p.L858R & 0.38 & 0.33 & NSCLC & 4 \\
\hline d024 & EGFR:p.L858R:c.2573T>G & 0.32 & 0.25 & NSCLC & - \\
\hline d025 & EGFR:exon20:c.C2369T:p.T790M & 0.32 & 0.31 & NSCLC & 4 \\
\hline d026 & EGFR exon18:c.2155G>T:p.G719C & 0.30 & 0.00 & Breast cancer & 3 \\
\hline d027 & EGFR:exon20:c.C2369T:p.T790M & 0.27 & 0.22 & NSCLC & 4 \\
\hline d028 & EGFR:exon20 c.C2369T:p.T790M & 0.25 & 0.22 & NSCLC & 4 \\
\hline d029 & EGFR:exon19:c.2236_2250del:p.746_750del & 0.24 & 0.34 & NSCLC & 3 \\
\hline d030 & KRAS:c.35G>A:p.G12D & 0.18 & 0.17 & NSCLC & 4 \\
\hline d031 & EGFR:exon20:c.C2369T:p.T790M & 0.16 & 0.00 & NSCLC & 4 \\
\hline d032 & EGFR:exon20:c.C2369T:p.T790M & 0.10 & 0.08 & NSCLC & - \\
\hline d033 & EGFR:exon20:c.C2369T:p.T790M & 0.09 & 0.04 & NSCLC & 4 \\
\hline d034 & EGFR:exon20:c.C2369T:p.T790M & 0.09 & 0.10 & NSCLC & 4 \\
\hline d035 & EGFR:exon20:c.C2369T:p.T790M & 0.07 & 0.03 & NSCLC & 4 \\
\hline
\end{tabular}

The mutant allele frequency of each variant detected in NGS and 3D Digital PCR. dPCR, 3D Digital PCR; NSCLC, non-small cell lung cancer are shown.

MAF variants, we used ARMS-PCR to test the 33 random selected FFPE samples with hotspot mutations detected by NGS and obtained a high concordance (PPV=100\%).

Taken together, we used high sequencing coverage and a statistical test with several hard filters generated from clinical samples to separate low MAF SNV/INDEL from false positives. To balance the cost of NGS and accuracy of variant calls for low MAF variants, we used pooled cell line models with certain germ line SNP in different data size to get the relationship accuracy between data size and variants. From this test, we validated the best target median coverage (2000X) that can meet the analysis requirement, whereas the low MAF variants detection needed to be corrected by dPCR. On the other hand, the overall performance of this assay was good in the ARMS-PCR test. However, our results cannot meet the requirement of different variant types in clinical use like other NGS-based approaches (17-20,39), which is one of the most important aspects for NGS compared to other traditional approaches. Furthermore, due to the DNA requirement of dPCR verification and quantity of extraction in plasma $(40,41)$, 
Table XIV. Summary of concordance between NGS and ARMS-PCR.

\begin{tabular}{|c|c|c|c|c|c|c|}
\hline Sample id & Mutation & NGS $(\%)$ & $\Delta \mathrm{Ct}$ & Results & Cancer type & Stage \\
\hline $\mathrm{a} 001$ & EGFR:exon20: c.C2369T: p.T790M & 2.00 & 6.64 & Positive & NSCLC & 4 \\
\hline $\mathrm{a} 002$ & EGFR: exon21: c.T2573G: p.L858R & 14.00 & 5.2 & Positive & NSCLC & 4 \\
\hline $\mathrm{a} 003$ & EGFR: exon21: c.T2573G: p.L858R & 14.00 & 4.47 & Positive & NSCLC & 4 \\
\hline $\mathrm{a} 004$ & EGFR: exon21: c.T2573G: p.L858R & 3.00 & 7.38 & Positive & NSCLC & 3 \\
\hline $\mathrm{a} 005$ & EGFR: exon21: c.T2573G: p.L858R & 4.00 & 6.01 & Positive & NSCLC & 4 \\
\hline $\mathrm{a} 006$ & EGFR: exon21: c.T2573G: p.L858R & 13.00 & 4.51 & Positive & NSCLC & 3 \\
\hline $\mathrm{a} 007$ & EGFR: exon21: c.T2573G: p.L858R & 9.00 & 5.45 & Positive & NSCLC & - \\
\hline $\mathrm{a} 008$ & EGFR: exon21: c.T2573G: p.L858R & 2.00 & 10.81 & Positive & NSCLC & 3 \\
\hline $\mathrm{a} 009$ & EGFR: exon21: c.T2573G: p.L858R & 16.00 & 3.96 & Positive & NSCLC & 4 \\
\hline $\mathrm{a} 010$ & EGFR: exon21: c.T2573G: p.L858R & 63.00 & 2.13 & Positive & NSCLC & 4 \\
\hline $\mathrm{a} 011$ & EGFR: exon21: c.T2573G: p.L858R & 31.00 & 2.32 & Positive & NSCLC & 4 \\
\hline $\mathrm{a} 012$ & EGFR: exon21: c.T2573G: p.L858R & 13.00 & 8.3 & Positive & NSCLC & 4 \\
\hline $\mathrm{a} 013$ & EGFR:exon21:c.T2582A:p.L861Q & 8.00 & 13.61 & positive & NSCLC & 4 \\
\hline $\mathrm{a} 014$ & EGFR:exon20:c.C2369T:p.T790M & 13.00 & 5.47 & Positive & NSCLC & 4 \\
\hline $\mathrm{a} 015$ & EGFR:exon19:c.2235_2249del:p.745_750del & 15.00 & 2.21 & Positive & NSCLC & - \\
\hline $\mathrm{a} 016$ & EGFR:exon19:c.2235_2249del:p.745_750del & 9.00 & 3.36 & Positive & NSCLC & 4 \\
\hline $\mathrm{a} 017$ & EGFR:exon19:c.2235_2249del:p.745_750del & 7.00 & 3.28 & Positive & NSCLC & 4 \\
\hline $\mathrm{a} 018$ & EGFR:exon19:c.2239_2256del:p.747_752del & 12.00 & 7.9 & Positive & NSCLC & 4 \\
\hline $\mathrm{a} 019$ & EGFR:exon19:c.2236_2250del:p.746_750del & 8.00 & 5.39 & Positive & NSCLC & 4 \\
\hline $\mathrm{a} 020$ & EGFR:exon19:c.2236_2250del:p.746_750del & 13.00 & 4.57 & Positive & NSCLC & 3 \\
\hline $\mathrm{a} 021$ & EGFR:exon19:c.2236_2250del:p.746_750del & 10.00 & 4.18 & Positive & NSCLC & 3 \\
\hline $\mathrm{a} 022$ & EGFR:exon19:c.2254_2277del:p.752_759del & 8.00 & 3.1 & Positive & NSCLC & 4 \\
\hline $\mathrm{a} 023$ & EGFR:exon19:c.2237_2254del:p.746_752del & 9.00 & 3.99 & Positive & NSCLC & 4 \\
\hline $\mathrm{a} 024$ & EGFR:exon19:c.2237_2254del:p.746_752del & 12.00 & 3.22 & Positive & NSCLC & 4 \\
\hline $\mathrm{a} 025$ & EGFR:exon19:c.2238_2252del:p.746_751del & 15.00 & 2.91 & Positive & NSCLC & - \\
\hline $\mathrm{a} 026$ & EGFR:exon19:c.2235_2249del:p.745_750del & 7.00 & 3.36 & Positive & NSCLC & 4 \\
\hline $\mathrm{a} 027$ & EGFR:exon19:c.2235_2249del:p.745_750del & 11.00 & 2.95 & Positive & NSCLC & 4 \\
\hline $\mathrm{a} 028$ & EGFR:exon19:c.2240_2254del:p.747_752del & 20.00 & 3.88 & Positive & NSCLC & 4 \\
\hline $\mathrm{a} 029$ & EGFR:exon19:c.2236_2250del:p.746_750del & 19.00 & 5.21 & Positive & NSCLC & 4 \\
\hline $\mathrm{a} 030$ & EGFR:exon19:c.2235_2249del:p.745_750del & 16.00 & 2.33 & Positive & NSCLC & 4 \\
\hline $\mathrm{a} 031$ & EGFR:exon19:c.2235_2249del:p.745_750del & 9.05 & 3.06 & Positive & NSCLC & - \\
\hline $\mathrm{a} 032$ & EGFR:exon19:c.2237_2253del:p.746_751del & 11.00 & 3.22 & Positive & NSCLC & 4 \\
\hline $\mathrm{a} 033$ & EGFR:exon19:c.2235_2249del:p.745_750del & 13.00 & 2.8 & Positive & NSCLC & 4 \\
\hline
\end{tabular}

The specificity of our assay in clinical samples. Thirty-three randomly selected FFPE tissues with positive detection in NGS were tested by ARMS-PCR. The $\Delta \mathrm{Ct}$ was the $\mathrm{Ct}$ value of sample minus control and the cut-off for T790M, L858R, L861Q, 19-Del were 8, 11, 12, 11, respectively. $\Delta \mathrm{Ct}, \mathrm{Ct}$ (sample) - $\mathrm{Ct}$ (control). NSCLC, non-small cell lung cancer.

this NGS-dPCR combined approach could only be used in FFPE sample but not plasma. With the advantages of noninvasive and overcome tumor-heterogeneity (42-44), the sequencing of plasma sample still needed more study. To reduce the sequencing errors confound with rare mutations, a NGS method termed Duplex sequencing was developed these years and may be useful in future plasma sequencing (45-47). In addition, given the capability of NGS test to detect variants with low MAF, the correlation between the NGS clinical report and the effect of targeted therapy still need further assessment (48). Finally, our NCP assay can give more mutation information and thus expand the treatment choices for patients, but more efforts still need to be done for future cancer diagnostics.

\section{References}

1. Renfro LA, An MW and Mandrekar SJ: Precision oncology: Anew era of cancer clinical trials. Cancer Lett S0304-3835(16)30163-X, 2016.

2. Arteaga CL and Baselga J: Impact of genomics on personalized cancer medicine. Clin Cancer Res 18: 612-618, 2012.

3. MacConaill LE, Van Hummelen P, Meyerson M and Hahn WC: Clinical implementation of comprehensive strategies to characterize cancer genomes: Opportunities and challenges. Cancer Discov 1: 297-311, 2011. 
4. Romano E, Schwartz GK, Chapman PB, Wolchock JD and Carvajal RD: Treatment implications of the emerging molecular classification system for melanoma. Lancet Oncol 12: 913-922, 2011.

5. Kwak EL, Bang YJ, Camidge DR, Shaw AT, Solomon B, Maki RG, Ou SH, Dezube BJ, Jänne PA, Costa DB, et al: Anaplastic lymphoma kinase inhibition in non-small-cell lung cancer. N Engl J Med 363: 1693-1703, 2010.

6. Shaw AT, Kim DW, Nakagawa K, Seto T, Crinó L, Ahn MJ, De Pas T, Besse B, Solomon BJ, Blackhall F, et al: Crizotinib versus chemotherapy in advanced ALK-positive lung cancer. N Engl J Med 368: 2385-2394, 2013.

7. Bollag G, Tsai J, Zhang J, Zhang C, Ibrahim P, Nolop K and Hirth P: Vemurafenib: The first drug approved for BRAF-mutant cancer. Nat Rev Drug Discov 11: 873-886, 2012.

8. Garraway LA and Lander ES: Lessons from the cancer genome. Cell 153: 17-37, 2013

9. Pao W: New approaches to targeted therapy in lung cancer. Proc Am Thorac Soc 9: 72-73, 2012.

10. Thomas RK, Baker AC, Debiasi RM, Winckler W, Laframboise T, Lin WM, Wang M, Feng W, Zander T, MacConaill L, et al: Highthroughput oncogene mutation profiling in human cancer. Nat Genet 39: 347-351, 2007.

11. MacConaill LE, Campbell CD, Kehoe SM, Bass AJ, Hatton C, Niu L, Davis M, Yao K, Hanna M, Mondal C, et al: Profiling critical cancer gene mutations in clinical tumor samples. PLoS One 4: e7887, 2009.

12. Tao YF, Wu D, Pang L, Zhao WL, Lu J, Wang N, Wang J, Feng X, Li YH, Ni J, et al: Analyzing the gene expression profile of pediatric acute myeloid leukemia with real-time PCR arrays. Cancer Cell Int 12: 1946-1958, 2012.

13. McCourt CM, Boyle D, James J and Salto-Tellez M: Immunohistochemistry in the era of personalised medicine. J Clin Pathol 66: 58-61, 2013.

14. Forbes SA, Bindal N, Bamford S, Cole C, Kok CY, Beare D, Jia M, Shepherd R, Leung K, Menzies A, et al: COSMIC: Mining complete cancer genomes in the Catalogue of Somatic Mutations in Cancer. Nucleic Acids Res 39 (Database issue): D945-D950, 2010.

15. Lawrence MS, Stojanov P, Mermel CH, Robinson JT, Garraway LA, Golub TR, Meyerson M, Gabriel SB, Lander ES and Getz G: Discovery and saturation analysis of cancer genes across 21 tumour types. Nature 505: 495-501, 2014.

16. Lawrence MS, Stojanov P, Polak P, Kryukov GV, Cibulskis K, Sivachenko A, Carter SL, Stewart C, Mermel CH, Roberts SA, et al: Mutational heterogeneity in cancer and the search for new cancer-associated genes. Nature 499: 214-218, 2013.

17. Frampton GM, Fichtenholtz A, Otto GA, Wang K, Downing SR, He J, Schnall-Levin M, White J, Sanford EM, An P, et al: Development and validation of a clinical cancer genomic profiling test based on massively parallel DNA sequencing. Nat Biotechnol 31: 1023-1031, 2013.

18. Hovelson DH, McDaniel AS, Cani AK, Johnson B, Rhodes K, Williams PD, Bandla S, Bien G, Choppa P, Hyland F, et al: Development and validation of a scalable next-generation sequencing system for assessing relevant somatic variants in solid tumors. Neoplasia 17: 385-399, 2015.

19. Choudhary A, Mambo E, Sanford T, Boedigheimer M, Twomey B, Califano J, Hadd A, Oliner KS, Beaudenon S, Latham GJ, et al: Evaluation of an integrated clinical workflow for targeted next-generation sequencing of low-quality tumor DNA using a 51-gene enrichment panel. BMC Med Genomics 7: 62, 2014.

20. Cheng DT, Mitchell TN, Zehir A, Shah RH, Benayed R, Syed A Chandramohan R, Liu ZY, Won HH, Scott SN, et al: Memorial Sloan Kettering-Integrated Mutation Profiling of Actionable Cancer Targets (MSK-IMPACT): A hybridization capturebased next-generation sequencing clinical assay for solid tumor molecular oncology. J Mol Diagn 17: 251-264, 2015.

21. Cibulskis K, McKenna A, Fennell T, Banks E, DePristo M and Getz G: ContEst: Estimating cross-contamination of human samples in next-generation sequencing data. Bioinformatics 27: 2601-2602, 2011 .

22. Gerlinger M, Rowan AJ, Horswell S, Larkin J, Endesfelder D, Gronroos E, Martinez P, Matthews N, Stewart A, Tarpey P, et al: Intratumor heterogeneity and branched evolution revealed by multiregion sequencing. N Engl J Med 366: 883-892, 2012.
23. Kinz E, Leiherer A, Lang AH, Drexel $\mathrm{H}$ and Muendlein A: Accurate quantitation of JAK2 V617F allele burden by arraybased digital PCR. Int J Lab Hematol 37: 217-224, 2015.

24. Shao D1, Lin Y, Liu J, Wan L, Liu Z, Cheng S, Fei L, Deng R, Wang J, Chen X, et al: A targeted next-generation sequencing method for identifying clinically relevant mutation profiles in lung adenocarcinoma. Sci Rep 6: 22338, 2016.

25. Forbes SA1, Tang G, Bindal N, Bamford S, Dawson E, Cole C, Kok CY, Jia M, Ewing R, Menzies A, et al: COSMIC (the Catalogue of Somatic Mutations in Cancer): A resource to investigate acquired mutations in human cancer. Nucleic Acids Res 8 (Database issue): D652-D657, 2009.

26. Abecasis GR, Altshuler D, Auton A, Brooks LD, Durbin RM, Gibbs RA, Hurles ME and McVean GA; 1000 Genomes Project Consortium: A map of human genome variation from populationscale sequencing. Nature 467: 1061-1073, 2010.

27. Warrick JI, Hovelson DH, Amin A, Liu CJ, Cani AK, McDaniel AS, Yadati V, Quist MJ, Weizer AZ, Brenner JC, et al: Tumor evolution and progression in multifocal and paired non-invasive/invasive urothelial carcinoma. Virchows Arch 466: 297-311, 2015.

28. Li H and Durbin R: Fast and accurate long-read alignment with Burrows-Wheeler transform. Bioinformatics 26: 589-595, 2010.

29. McKenna A, Hanna M, Banks E, Sivachenko A, Cibulskis K, Kernytsky A, Garimella K, Altshuler D, Gabriel S, Daly M, et al: The Genome Analysis Toolkit: A MapReduce framework for analyzing next-generation DNA sequencing data. Genome Res 20: 1297-1303, 2010

30. DePristo MA, Banks E, Poplin R, Garimella KV, Maguire JR, Hartl C, Philippakis AA, del Angel G, Rivas MA, Hanna M, et al: A framework for variation discovery and genotyping using next-generation DNA sequencing data. Nat Genet 43: 491-498, 2011.

31. Li H, Handsaker B, Wysoker A, Fennell T, Ruan J, Homer N, Marth G, Abecasis G and Durbin R; 1000 Genome Project Data Processing Subgroup: The sequence alignment/map format and SAMtools. Bioinformatics 25: 2078-2079, 2009.

32. Arsenic R, Treue D, Lehmann A, Hummel M, Dietel M, Denkert $C$ and Budczies J: Comparison of targeted nextgeneration sequencing and Sanger sequencing for the detection of PIK3CA mutations in breast cancer. BMC Clin Pathol 15: 20 , 2015.

33. Borad MJ, Champion MD, Egan JB, Liang WS, Fonseca R, Bryce AH, McCullough AE, Barrett MT, Hunt K, Patel MD, et al: Integrated genomic characterization reveals novel, therapeutically relevant drug targets in FGFR and EGFR pathways in sporadic intrahepatic cholangiocarcinoma. PLoS Genet 10: e1004135, 2014.

34. Hadd AG, Houghton J, Choudhary A, Sah S, Chen L, Marko AC, Sanford T, Buddavarapu K, Krosting J, Garmire L, et al: Targeted, high-depth, next-generation sequencing of cancer genes in formalin-fixed, paraffin-embedded and fine-needle aspiration tumor specimens. J Mol Diagn 15: 234-247, 2013.

35. Roychowdhury S, Iyer MK, Robinson DR, Lonigro RJ, Wu YM, Cao X, Kalyana-Sundaram S, Sam L, Balbin OA, Quist MJ, et al: Personalized oncology through integrative high-throughput sequencing: A pilot study. Sci Transl Med 3: 111ra121, 2011.

36. Kerick M, Isau M, Timmermann B, Sültmann H, Herwig R, Krobitsch S, Schaefer G, Verdorfer I, Bartsch G, Klocker H, et al: Targeted high throughput sequencing in clinical cancer settings: Formaldehyde fixed-paraffin embedded (FFPE) tumor tissues, input amount and tumor heterogeneity. BMC Med Genomics 4: 68, 2011.

37. Schweiger MR, Kerick M, Timmermann B, Albrecht MW, Borodina T, Parkhomchuk D, Zatloukal K and Lehrach $\mathrm{H}$ : Genome-wide massively parallel sequencing of formaldehyde fixed-paraffin embedded (FFPE) tumor tissues for copy-numberand mutation-analysis. PLoS One 4: e5548, 2009.

38. Chevrier S, Arnould L, Ghiringhelli F, Coudert B, Fumoleau P and Boidot R: Next-generation sequencing analysis of lung and colon carcinomas reveals a variety of genetic alterations. Int $\mathbf{J}$ Oncol 45: 1167-1174, 2014.

39. Cottrell CE, Al-Kateb H, Bredemeyer AJ, Duncavage EJ, Spencer DH, Abel HJ, Lockwood CM, Hagemann IS, O'Guin SM, Burcea LC, et al: Validation of a next-generation sequencing assay for clinical molecular oncology. J Mol Diagn 16: 89-105, 2014.

40. Haber DA and Velculescu VE: Blood-based analyses of cancer: Circulating tumor cells and circulating tumor DNA. Cancer Discov 4: 650-661, 2014. 
41. Arnedos M, Vicier C, Loi S, Lefebvre C, Michiels S, Bonnefoi H and Andre F: Precision medicine for metastatic breast cancer-limitations and solutions. Nat Rev Clin Oncol 12: 693-704, 2015.

42. Newman AM, Bratman SV, To J, Wynne JF, Eclov NC, Modlin LA, Liu CL, Neal JW, Wakelee HA, Merritt RE, et al: An ultrasensitive method for quantitating circulating tumor DNA with broad patient coverage. Nat Med 20: 548-554, 2014.

43. Ignatiadis M and Dawson SJ: Circulating tumor cells and circulating tumor DNA for precision medicine: Dream or reality? Ann Oncol 25: 2304-2313, 2014.

44. Lipson EJ, Velculescu VE, Pritchard TS, Sausen M, Pardoll DM, Topalian SL and Diaz LA Jr: Circulating tumor DNA analysis as a real-time method for monitoring tumor burden in melanoma patients undergoing treatment with immune checkpoint blockade. J Immunother Cancer 2: 42, 2014

45. Schmitt MW, Kennedy SR, Salk JJ, Fox EJ, Hiatt JB and Loeb LA: Detection of ultra-rare mutations by next-generation sequencing. Proc Natl Acad Sci USA 109: 14508-14513, 2012.
46. Kennedy SR, Schmitt MW, Fox EJ, Kohrn BF, Salk JJ, Ahn EH, Prindle MJ, Kuong KJ, Shen JC, Risques RA, et al: Detecting ultralow-frequency mutations by Duplex Sequencing. Nat Protoc 9: 2586-2606, 2014.

47. Newman AM, Lovejoy AF, Klass DM, Kurtz DM, Chabon JJ, Scherer F, Stehr H, Liu CL, Bratman SV, Say C, et al: Integrated digital error suppression for improved detection of circulating tumor DNA. Nat Biotechnol 34: 547-555, 2016.

48. Luo H, Li H, Hu Z, Wu H, Liu C, Li Y, Zhang X, Lin P, Hou Q, Ding $\mathrm{G}$, et al: Noninvasive diagnosis and monitoring of mutations by deep sequencing of circulating tumor DNA in esophageal squamous cell carcinoma. Biochem Biophys Res Commun 471: 596-602, 2016 